\title{
A DNA-origami nuclear pore mimic reveals nuclear entry mechanisms of HIV-1 capsid
}

Qi Shen, ${ }^{1,2,3}$ Chaoyi Xu, ${ }^{4}$ Sooin Jang, ${ }^{5,6}$ Qiancheng Xiong, ${ }^{2,3}$ Swapnil C. Devarkar, ${ }^{1}$ Taoran Tian, ${ }^{3}$ Gregory J. Bedwell, ${ }^{5,6}$ Therese N. Tripler, ${ }^{1}$ Yingxia Hu, ${ }^{1}$ Shuai Yuan, ${ }^{1}$ Joshua Temple, ${ }^{1}$ Jiong Shi, ${ }^{7}$ Christopher Aiken, ${ }^{7}$ Alan N. Engelman, ${ }^{5,6}$ Juan R. Perilla, ${ }^{4}$ C. Patrick Lusk, ${ }^{2}$ Chenxiang Lin, ${ }^{2,3,}{ }^{*}$ and Yong Xiong ${ }^{1,8, *}$

${ }^{1}$ Department of Molecular Biophysics and Biochemistry, Yale University, New Haven, CT, 06511, USA

${ }^{2}$ Department of Cell Biology, Yale School of Medicine, New Haven, CT, 06520, USA ${ }^{3}$ Nanobiology Institute, Yale University, West Haven, CT, 06516, USA

${ }^{4}$ Department of Chemistry and Biochemistry, University of Delaware, Newark, DE, 19716, USA

${ }^{5}$ Department of Cancer Immunology and Virology, Dana-Farber Cancer Institute, Boston, MA, 02215, USA

${ }^{6}$ Department of Medicine, Harvard Medical School, Boston, MA, 02115, USA

${ }^{7}$ Department of Pathology, Microbiology and Immunology, Vanderbilt University Medical Center, Nashville, TN, 37232, USA

${ }^{8}$ Lead Contact

*Correspondence: chenxiang.lin@yale.edu (C.L.), yong.xiong@yale.edu (Y.X.) 


\section{Summary}

The capsid of human immunodeficiency virus 1 (HIV-1) plays a pivotal role in viral nuclear import, but the mechanism by which the viral core passages the nuclear pore complex (NPC) is poorly understood. Here, we use DNA-origami mimics of the NPC, termed NuPODs (NucleoPorins Organized by DNA), to reveal the mechanistic underpinnings of HIV-1 capsid nuclear entry. We found that trimeric interface formed via three capsid protein hexamers is targeted by a triple-arginine (RRR) motif but not the canonical phenylalanine-glycine (FG) motif of NUP153. As NUP153 is located on the nuclear face of the NPC, this result implies that the assembled capsid must cross the NPC in vivo. This hypothesis is corroborated by our observations of tubular capsid assemblies penetrating through NUP153 NuPODs. NUP153 prefers to bind highly curved capsid assemblies including those found at the tips of viral cores, thereby facilitating capsid insertion into the NPC. Furthermore, a balance of capsid stabilization by NUP153 and deformation by CPSF6, along with other cellular factors, may allow for the intact capsid to pass NPCs of various sizes. The NuPOD system serves as a unique tool for unraveling the previously elusive mechanisms of nuclear import of HIV-1 and other viruses.

\section{Keywords}

HIV-1 capsid, nuclear entry, DNA-origami, nuclear pore mimic, RRR-motif, pattern sensing, curvature preference, capsid stabilization, capsid deformation

\section{Introduction}

As a retrovirus, HIV-1 reverse-transcribes its RNA genome for integration into host chromatin within the nucleus (Lusic and Siliciano, 2017). However, it remains unclear how HIV-1 gains access to the nuclear compartment, which is segregated from the cytoplasm by a double membraned nuclear envelope. The most logical hypothesis is that HIV-1 passes through nuclear pore complexes (NPCs), which are massive protein assemblies embedded in the nuclear envelope that control molecular exchange between the nucleus and the cytoplasm. Indeed, recent evidence suggests that the intact HIV-1 capsid is capable of crossing the NPC and disassembles only in the nuclear interior (Burdick et al., 2020). However, this is conceptually challenging, given that the commonly accepted $\sim 40 \mathrm{~nm}$ diameter of the NPC central transport channel (von Appen et al., 2015) is smaller than the $\sim 60 \mathrm{~nm}$ width of an intact capsid (Briggs et al., 2003; Mattei et al., 2016). While recent data supports that there may be energy-dependent changes in the NPC scaffold that might dilate the channel (Zimmerli et al., 2020), the channel itself is also filled with dozens of intrinsically disordered proteins (nucleoporins/NUPs) rich in repeating FG motifs, which establish a diffusion barrier for macromolecules. The mechanism by which a largely intact HIV1 capsid passes through the NPC central channel remains to be elucidated. 
HIV-1 capsid is composed of capsomeres of approximately 250 capsid protein (CA) hexamers and 12 CA pentamers, which assemble into a fullerene cone (Briggs et al., 2003; Ganser et al., 1999; Li et al., 2000; Mattei et al., 2016; Pornillos et al., 2011). The capsid furnishes an enclosed compartment necessary for reverse transcription and concomitantly protects the genome from destruction by host immune surveillance and cellular antiviral restriction factors (Hulme et al., 2015; Hulme et al., 2011; Yamashita and Engelman, 2017). Most capsid-targeting host factors sense higher-order patterns formed by multiple capsomeres, and in vitro assembly techniques have matured in recent years to capture such patterns. CA can be assembled through various disulfide bonds into standalone hexamers or pentamers (Pornillos et al., 2009; Pornillos et al., 2011), or multi-hexamer/pentamer units as well as a hexamer- 2 structure that mimics the interface of three hexamers in the assembled capsid (Summers et al., 2019). Further assembly of higher-order structures such as CA tubes (Lopez et al., 2011), spheres (Zhang et al., 2018), and stable cones are also achievable (Dick et al., 2018) (Figure 1A). However, these assemblies have not yet been applied to exploring interactions with the NPC as there are few in vitro platforms that can mimic the dimensions and complexity of the NPC.

At $\sim 100 \mathrm{MDa}$, the NPC is the largest macromolecular complex in the human cell, which is built from $~ 30$ different NUPs each in 16 or 32 copies (Brohawn et al., 2009; Cook et al., 2007; Lin and Hoelz, 2019a; Strambio-De-Castillia et al., 2010). Many FGrich NUPs can interact with HIV-1 capsid, including NUP358, NUP214, NUP88, NUP62, and NUP153 (Bichel et al., 2013; Dharan et al., 2020; Di Nunzio et al., 2013; Kane et al., 2018; Matreyek and Engelman, 2011). Apart from these NUPs, soluble nuclear transport receptors (NTRs) including Importin $\alpha 3$ (Ao et al., 2010), Karyopherin B2/Transportin-1 (TRN-1) (Fernandez et al., 2019), and Transportin-3 (TRN-SR2) (Maertens et al., 2014; Valle-Casuso et al., 2012) are implicated in HIV-1 nuclear entry. Other host factors, including cyclophilin A (CypA) (Schaller et al., 2011; Yamashita and Engelman, 2017), cleavage and polyadenylation specificity factor 6 (CPSF6) (Achuthan et al., 2018; Bejarano et al., 2019; Bhattacharya et al., 2014; Chin et al., 2015; Price et al., 2012; Sowd et al., 2016), and myxovirus resistance protein B (MxB) (Busnadiego et al., 2014; Kane et al., 2018; Kane et al., 2013; King et al., 2004), can bind the capsid and affect its nuclear transport. This complexity limits the impact of cellular methods such as targeted NUP knockdowns to reveal capsid transport mechanisms (Kane et al., 2018).

To establish a simplified model system that circumvents these difficulties, we took advantage of our prior generation of the NucleoPorins Organized by DNA (NuPOD) platform (Fisher et al., 2018). This experimental system leverages the DNAorigami technique (Dietz et al., 2009; Rothemund, 2006; Seeman and Sleiman, 2018) to assemble a DNA ring that mimics the $\sim 40 \mathrm{~nm}$ diameter of the NPC transport channel (Fisher et al., 2018; Ketterer et al., 2018; von Appen et al., 2015). Multiple copies of multiple NUPs can be attached to the DNA-origami rings at specific 
positions, with additional tunable parameters such as protein stoichiometry, grafting density, and topology (Fisher et al., 2018; Ketterer et al., 2018). Therefore, our NuPOD system is ideally suited to interrogate the involvement of individual or specific combinations of NUPs in HIV-1 capsid nuclear import. Here we have focused on NUP153, a component of the NPC's nuclear basket (Schmitz et al., 2010; Walther et al., 2001), because it binds capsid directly and is essential for HIV-1 capsid nuclear transport (Matreyek and Engelman, 2011; Price et al., 2014). Since NUP153 resides in the nuclear side of the NPC (Buffone et al., 2018; Duheron et al., 2014), the capsid would need to insert deep into or pass through the NPC to reach it. Furthermore, as NUP153 plays an important role in the late stage of capsid nuclear transport, understanding its actions is key to unraveling the nuclear entry mechanisms of HIV-1 (Chen et al., 2016; Matreyek and Engelman, 2011; Matreyek et al., 2013).

In this work, we combined NuPOD with capsid engineering techniques to reveal the mechanistic underpinnings of capsid nuclear import in vitro. We identified the mode of capsid pattern-sensing by the NUP153 C-terminal tail, via an RRR-motif but not the canonical FG-motif, which strongly binds higher-order capsid structures beyond single hexamers and in doing so stabilizes the CA lattice. We created NuPODs with 32 copies of the NUP153 C-terminal domain (residues 896-1475; NUP153CTD), the endogenous number in the NPC (Ori et al., 2013), to simulate the critical features of the natural nuclear pore environment. Capsids isolated from HIV-1 virions and in vitro assembled structures bind these NUP153 NuPODs in a shape and curvature dependent manner. Strikingly, tubular CA assemblies can thread through the central pore of the NUP153 NuPOD, strongly supporting the transport of the assembled viral capsid through the nuclear pore.

\section{Results}

\section{NUP153 targets lattice interfaces of the assembled capsid}

It is well established that NUP153 directly interacts with the capsid and is essential for HIV-1 nuclear import. NUP153 is composed of 1475 amino acids (aa) and contains 24 FG repeats in its CTD (Figure 1B) (Fahrenkrog et al., 2002; Matreyek et al., 2013). While the CA hexamer contains sites for general FG-motif binding, it has been shown that only the last FG-motif in the C-terminal tail of NUP153 was needed for the capsid interaction (Matreyek et al., 2013). Consistently, crystal structures showed that the FG-motif in the NUP153 C-terminal tail binds at the interface between two subunits within the CA hexamer, albeit with a relatively weak affinity ( $K_{d}$ of $\sim 50 \mu \mathrm{M}$ ) (Buffone et al., 2018; Price et al., 2014). 

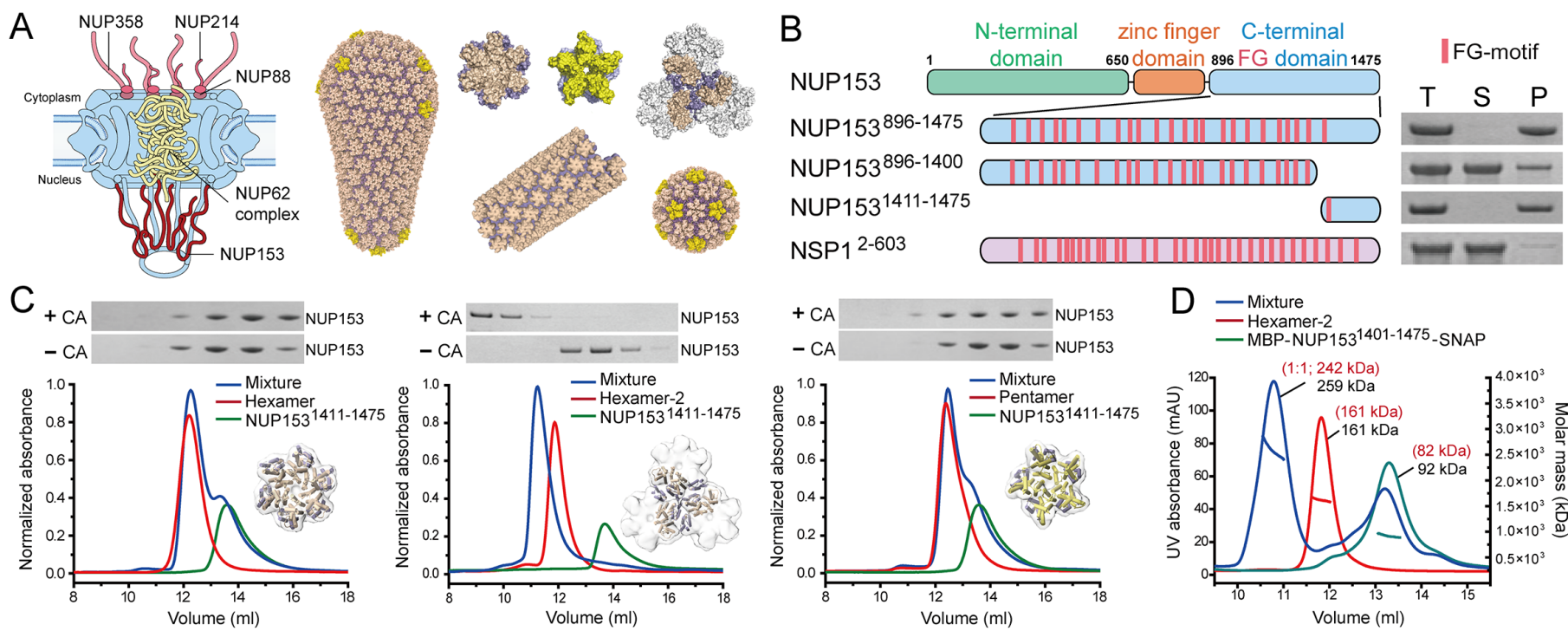

Figure 1. NUP153 targets the lattice interfaces in the assembled capsid.

(A) Schematic of the NPC with NUPs that have been shown to bind to HIV-1 capsid (left) and various in vitro assembled CA structures used in this study (right).

(B) Co-pelleting assay of NUP153 CTD variants and yeast nucleoporin NSP1 control with CA tubes. Left: Schematics of the constructs with FG repeats in each protein indicated as red bars. Right: The Total ( $T$ ), Soluble (S), and Pellet (P) fractions of co-pelleting assays using A14C/E45C disulfide crosslinked CA tubes analyzed by SDS-PAGE.

(C) Size-exclusion chromatography co-elution assays of CA hexamer (left), hexamer-2 (middle) or pentamer (right) with NUP153 $3^{1411-1475}$. Top: SDS-PAGE of eluates. Only hexamer-2 induced a peak shift of NUP153 ${ }^{1411-1475}$, indicating co-elution, with a corresponding shift of the elution fraction bands in SDS-PAGE (top).

(D) SEC-MALS analysis of NUP153 $3^{1401-1475}$ and hexamer-2. Overlay of the individual SEC-MALS elution profiles of NUP153 ${ }^{1401-1475}$ (red), hexamer-2 (green), and a mixture of the two (blue). The measured (black) and predicted (red) molecular weights are shown.

See also Figure S1.

We tested the interaction between purified NUP153 constructs and nanotubes assembled from purified CA protein in a co-pelleting assay (Figure $1 \mathrm{~B}$ and $\mathrm{S} 1$ ). For this purpose, $A 14 C / E 45 C$ disulfide cross-linked CA tubes were employed due to their known stability under low salt conditions (Lopez et al., 2011). Consistent with previous findings, the C-terminal tail (residues 1411-1475, NUP153 ${ }^{1411-1475}$ ) efficiently bound to CA tubes. The preceding NUP153 region (residues 896-1410 with 23 FG-repeats) or control NUP, yeast nucleoporin NSP1 (S. cerevisiae orthologue of NUP62 with 32 FG-repeats) showed little or no binding, respectively. These results, together with the reported low binding affinity of the NUP153 FG-containing peptide to CA hexamers $\left(K_{d} \sim 50 \mu \mathrm{M}\right)$, highlights that FG motifs have limited inherent capsidbinding activity. By contrast, we observed complete co-pelleting of NUP153 $3^{1411-1475}$ with CA tubes even at comparatively low NUP concentrations ( $3 \mu \mathrm{M}$ ) (Figure $1 \mathrm{~B}$ and S1). Since the CA tubes present the higher-order lattice of the assembled capsid, it suggests the existence of a strong interaction beyond the hexamer-specific NUP153 FG-motif binding site. 
To provide a more detailed understanding of how NUP153 binds HIV-1 capsid, we analyzed the interaction between NUP153 ${ }^{1411-1475}$ and various CA assemblies representing different patterns on the capsid. We performed size exclusion chromatography (SEC) analysis of NUP153 ${ }^{1411-1475}$ with individual capsomeres (CA hexamers or pentamers), as well as the hexamer- 2 assembly that mimics the interface between 3 hexamers (Summers et al., 2019) (Figure 1C). Consistent with the reported low binding affinities (Price et al., 2014), neither CA hexamer nor pentamer detectably bound NUP153 $3^{1411-1475}$. By contrast, efficient binding of hexamer-2 to NUP153 ${ }^{1411-1475}$ was readily detected (Figure $1 C$ ). We further used SECMALS (SEC-multiangle light scattering) to measure the molecular weight distributions in each elution peak and deduce the stoichiometric ratios of NUP153 and hexamer-2 in the complex. The results showed good agreement with a 1:1 molar ratio of NUP1531401-1475: hexamer-2 (Figure 1D), pointing to a model of the C-terminal tail of NUP153 engaging inter-hexamer interfaces on the capsid. These observations revealed that NUP153 preferentially engages the higher-order lattice within the assembled capsid, implying that capsid interaction with the NPC requires at least a partially assembled capsid.

\section{The positively charged C-terminal tail of NUP153 binds to the 3-fold tri-hexamer interface in capsid}

The higher-order capsid lattice preference of NUP153 ${ }^{1411-1475}$ suggested it contains a major binding site beyond the CA hexamer-targeting FG-motif. We performed a comprehensive mapping study to identify the specific region responsible for highaffinity binding with the capsid (Figure $2 \mathrm{~A}$ and S2). Substantial binding (60\%-75\%) remained with deletion or truncation of the FG-motif (NUP153 ${ }^{1401-1475 F T F G \Delta}$ or NUP153 ${ }^{1425-1475}$ ), supporting the notion that the FG-motif provides some but not the majority of the binding affinity (Figure $2 \mathrm{~A}$ and $\mathrm{S} 2$ ). When the $\mathrm{N}$-terminal region of the NUP153 C-terminal tail was progressively removed (NUP153 ${ }^{1436-1475}$ ), a moderate further decrease in CA tube binding was observed. Interestingly, even the shortest Cterminal tail construct, containing only the last 11 aa of the protein (NUP153 ${ }^{1465-1475}$ ), retained close to $50 \%$ binding. On the other hand, removal of the last 11 aa region (NUP153 ${ }^{1411-1465}$ ) retained only $25 \%$ of the binding. These results indicated that the C-terminal 11 residues of NUP153 contain a strong capsid-binding site (Figure 2A and S2).

The short 11 aa C-terminal tail of NUP153 is enriched in positively-charged amino acids, including a triple-arginine (RRR) motif that bears resemblance to the capsid-binding motif in MxB (Busnadiego et al., 2014; Kane et al., 2018; Kane et al., 2013; King et al., 2004). Since the MxB RRR-motif targets capsid through electrostatic interactions, we tested the effect of increasing salt concentrations on CA tube binding. Indeed, when the $\mathrm{NaCl}$ concentration was increased from $75 \mathrm{mM}$ to 300 mM, NUP153 $3^{1411-1475}$ lost over half of its binding capacity, while NUP153 ${ }^{1411-1464}$ containing the FG-motif but not the 11 aa tail showed no change in its binding 
(Figure $2 \mathrm{~A}$ and $\mathrm{S2}$ ). This salt sensitivity suggests that the RRR-motif engages the capsid in an MxB-like manner, likely binding at the negatively charged tri-hexamer center.

To test the hypothesis that NUP153 binds the capsid in a bipartite mode using both the FG-motif and the RRR-motif, we created a construct connecting the FGmotif to the last 11 aa region (NUP153 ${ }^{1411-1475 / 1425-1464 \Delta}$ ). The resultant 25 aa segment bound CA nanotubes as efficiently as the NUP153CTD (NUP153896-1475) did, confirming these two regions provide most of the capsid-binding capacity. We further used isothermal titration calorimetry (ITC) to quantify the binding affinity of this 25 aa peptide to the lattice-mimicking hexamer-2. This minimal NUP153 CTD peptide bound to hexamer- 2 with a $K_{\mathrm{d}}$ of $\sim 0.8 \mu \mathrm{M}$, which is close to two orders of magnitude tighter than the $K_{d}$ of $\sim 50 \mu \mathrm{M}$ reported for FG-hexamer binding (Price et al., 2014) (Figure 2B).

To confirm the results of in vitro binding assays under HIV-1 infection conditions, we employed a previously-described tripartite motif (TRIM) construct wherein the Cterminal domain of NUP153 ${ }^{896-1475}$ was fused to the RING, B-box 2, and coiled coil domains of rhesus TRIM5 $\alpha$ (Matreyek et al., 2013). Effector functions provided by the TRIM domains coupled with capsid binding, which was provided by the NUP153CTD, significantly inhibited HIV-1 infection (Matreyek et al., 2013) (Figure 2C). Deletion mutagenesis revealed that TRIM-NUP153 ${ }^{1401-1475}$ lacking the $505 \mathrm{~N}$ terminal residues of $896-1475$, as well as a minimal fusion construct that harbored just the 25 residues composed of the FG-motif (1411-1424) and RRR-motif (14651475), conferred full restriction activity (Figure 2C). These results support the findings from biochemical studies and confirm that NUP153 associates with the capsid via two regions: the first is the previously reported weaker FG-hexamer interface; the second is the positively charged RRR-motif that binds strongly (Figure $2 A, F)$.

To identify the capsid surface that is targeted by the RRR-motif of NUP153, we mutated key CA residues that are located in different regions of the capsid, including the tri-hexamer interface (EE212/213AA, E75A), the di-hexamer interface (EE180/181AA), the FG pocket (N74D, Q67A, N57D), the CypA-binding loop (P90A, G89V), and the hexamer central pore (R18D) (Bhattacharya et al., 2014; Dick et al., 2018; Price et al., 2014; Schaller et al., 2011; Smaga et al., 2019). Co-pelleting results showed that alterations of key residues at the tri-hexamer interface significantly reduced NUP153 binding to CA nanotubes (Figure 2D). By contrast, mutations in the di-hexamer interface, the CypA-binding loop, the FG-binding pocket, and the hexamer central pore showed little or no effect on binding (Figure 2D). These data again demonstrate the strong affinity of NUP153 for binding the CA lattice beyond just the hexamer. Similar to MxB's interaction with the CA lattice, the main driving force of association is the electrostatic contact between the positively charged RRR motif and the negatively charged CA tri-hexamer interface (Smaga et al., 2019). 
We next performed all-atom molecular dynamics (MD) simulations to gain a mechanistic understanding of the NUP153 CTD-CA interactions at the tri-hexamer interface. A NUP153 $3^{1451-1475}$ and hexamer- 2 complex model was built and simulated for $8.7 \mu \mathrm{s}$. The results of this MD simulation are shown in Figure S3 and Table S1. NUP153 $3^{1451-1475}$ was found to be mostly unstructured during the simulations (Figure S3A), except for a short $\alpha$-helix at the C-terminus (residue 1470 to 1475) that appeared temporarily (Figure S3C and Movie S1). The interaction between NUP153 $3^{1451-1475}$ and hexamer-2 appeared to be dynamic, rapidly transitioning between bound and unbound states as analyzed by the Markov state model (Chodera and Noe, 2014; Husic and Pande, 2018a; Wang et al., 2018b) (Figure S3). The NUP153 C-terminal tail formed high occupancy contacts with polar CA residues in different states (Table S1) whereby in the last state the RRR motif inserted deeply into the tri-hexamer interface, surrounded by negatively charged glutamate residues from 4 different CA monomers (Figure 2E, bottom).

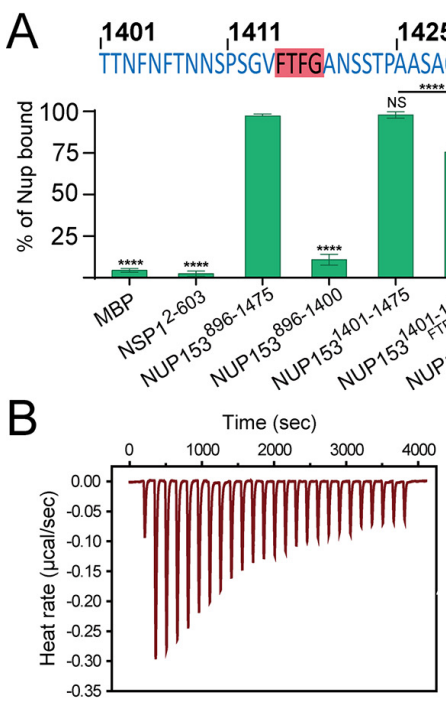

C

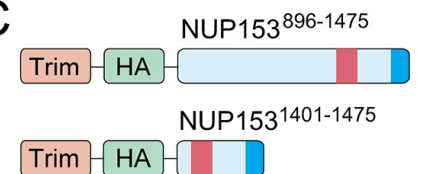

Trim HA \begin{tabular}{l}
1436 \\
1465 \\
1405 \\
\hline
\end{tabular}

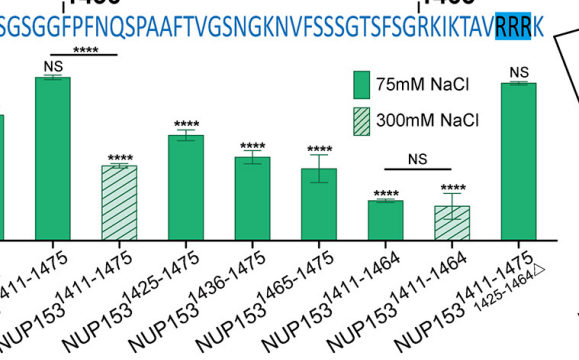

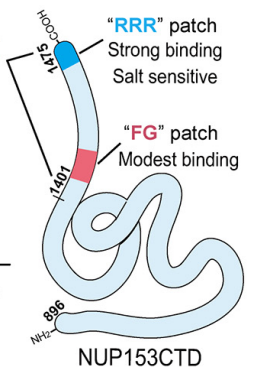

D

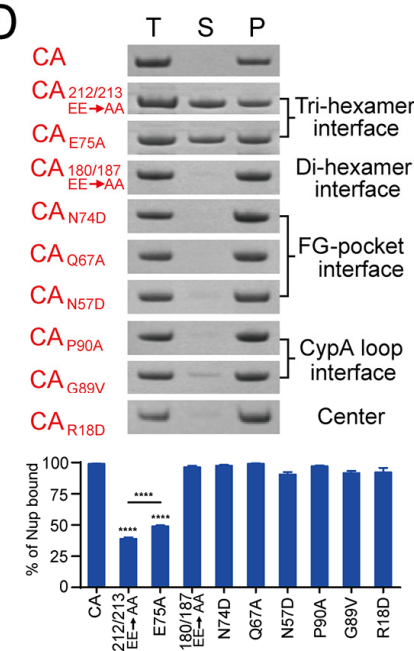

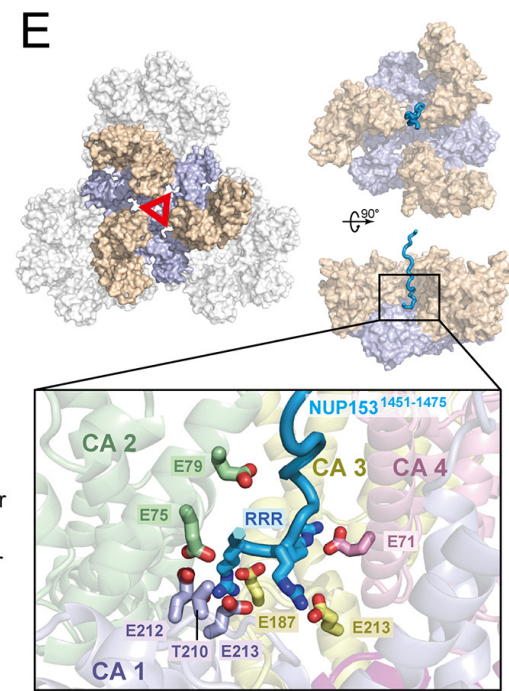

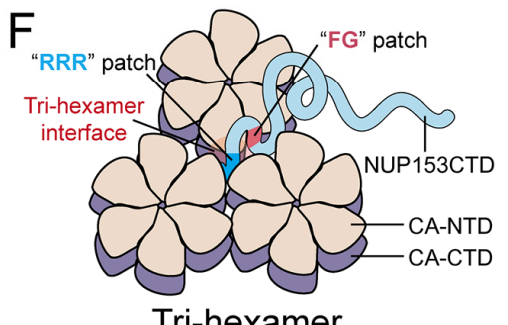

Tri-hexamer

Figure 2. A bipartite interaction between NUP153 and CA lattice.

(A) Top: The C-terminal 75 aa of NUP153. Bottom: percentages of various NUP153 variants, along with maltose-binding protein (MBP) and yeast nucleoporin NSP1 negative controls, bound to CA tubes in the co-pelleting assays under either low salt ( $75 \mathrm{mM} \mathrm{NaCl}$, solid green bar) or high salt (300 mM NaCl, striped green bar) conditions. Data were expressed as mean \pm standard deviation. Differences were tested by two-tailed Student's t-test. Compared with NUP153 ${ }^{894-1475}$; NS, not significant $(P \geqslant 0.05) ;{ }^{* * * *}, P<0.0001$. The schematic of the two capsid-binding motifs on NUP153CTD is shown in the right panel. 
(B) The NUP153 ${ }^{1411-1475 / 1425-1464 \triangle}$ peptide (25 aa) binds to CA hexamer-2 with $0.8 \pm 0.2 \mu \mathrm{M} K_{d}$ as measured by ITC.

(C) Restriction of HIV-1 infection by TRIM-NUP153 fusion proteins. Schematic of test constructs (left panel) and results (avg. \pm SD of two independent experiments) of HIV-1 infection assays (right panel) are shown. EV, empty vector. Statistical significance was determined by one-tailed Student's t-test; NS, not significant $(P \geq 0.05) ;{ }^{*}, P<0.05 ; * * *, P<0.001 ; * * * *, P<0.0001$. Immunoblot of protein expression is shown in Figure S2C.

(D) Co-pelleting assays of CA mutants with NUP153 ${ }^{1411-1475}$. Top: SDS-PAGE analysis of co-pelleted NUP153 ${ }^{1411-1475}$. Bottom: quantification of bound NUP153 $3^{1411-1475}$ normalized to that of wild-type CA tubes. Data were expressed as mean \pm standard deviation. Differences were tested by twotailed Student's t-test. Compared with A14C/E45C CA tubes; $* * * *, P<0.0001$.

(E) All-atom molecular dynamic simulations identify NUP153CTD interactions at the CA trihexamer center. Top: surface representation of the hexamer-2 tri-hexamer center region (left) and simulated NUP153 ${ }^{1451-1475}$ (light blue cartoon) binding to hexamer-2 (right). Bottom: the RRRmotif is surrounded by negatively charged glutamate residues (sticks) from 4 CA monomers (bottom).

(F) Schematics of the bipartite interaction between NUP153CTD and the CA tri-hexamer. See also Figure S2, Figure S3, Table S1, and Movie S1.

\section{Building a DNA-origami mimic of NUP153 organization within the human NPC} To interrogate capsid-NUP153 interaction in a scenario resembling the natural environment, we generated NuPODs with NUP153CTD attached to the inner surface of the $45 \mathrm{~nm}$ diameter DNA-origami ring (Fisher et al., 2018). The inner side of the ring has 32 single-stranded DNA "handles", to which complementary DNA oligonucleotides (anti-handles) conjugated to NUP153CTD can hybridize. We constructed 32 handles inside the NuPOD ring since there are 32 copies of NUP153 in the human NPC (Lin and Hoelz, 2019b; Ori et al., 2013). NUP153CTD was fused to a SNAP-tag, which reacts with a benzylguanine-labeled DNA oligo (BG-oligo) to covalently link the anti-handle to the protein. The SNAP-tag/BG-oligo reaction was very efficient, yielding more than $95 \%$ of NUP153CTD conjugation with anti-handles (Figure 3A). NUP153CTD-conjugated anti-handles were then hybridized with complementary handles at the inside of the origami ring to produce the NUP153 NuPOD, which was then purified by rate-zonal centrifugation (Lin et al., 2013). We used negative-stain electron microscopy (EM) to confirm the NUP153 NuPOD construction, which was characterized by NUP153CTD filling the origami ring interior (Figure 3B and S4).

\section{NUP153 NuPOD binds to the tip regions of in vitro assembled capsids and native viral cores.}

We utilized the NUP153 NuPOD to gain mechanistic insights into the NPC engagement of the cone-shaped HIV-1 capsid. We first tested its interaction with in vitro assembled CA cones (average length $\sim 120 \mathrm{~nm}$, width $\sim 60 \mathrm{~nm}$ ), which were produced using the A14C/E45C CA mutant in the presence of inositol hexakisphosphate (IP6) and CypA (Dick et al., 2018). As expected, the A14C/E45C 
crosslinked cones remained stable under low salt conditions (Figure S4). As visualized by negative-stain EM, the cones readily bound the NUP153 NuPODs. Under these conditions, the broad or narrow curved tip regions of the capsid cones were found to exclusively insert into the NUP153 NuPODs. We did not observe any NuPOD attachment to the flat surface of the capsid cone. NuPODs assembled with yeast nucleoporin NSP1 served as a negative control. Consistent with its lack of co-pelleting with CA nanotubes (Figure 2A), capsid cones failed to bind the yeast nucleoporin NSP1 NuPODs (Figure 3C, top right).

A

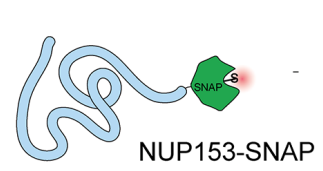

Benzylguanine-oligo
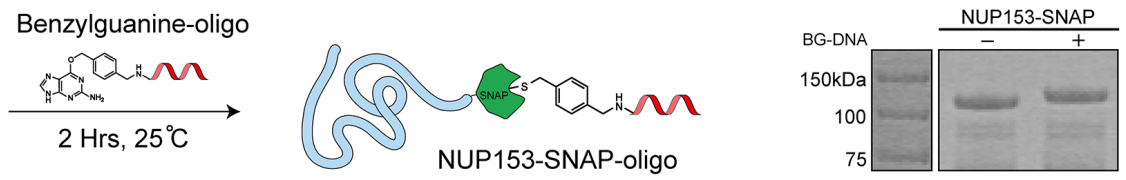

B
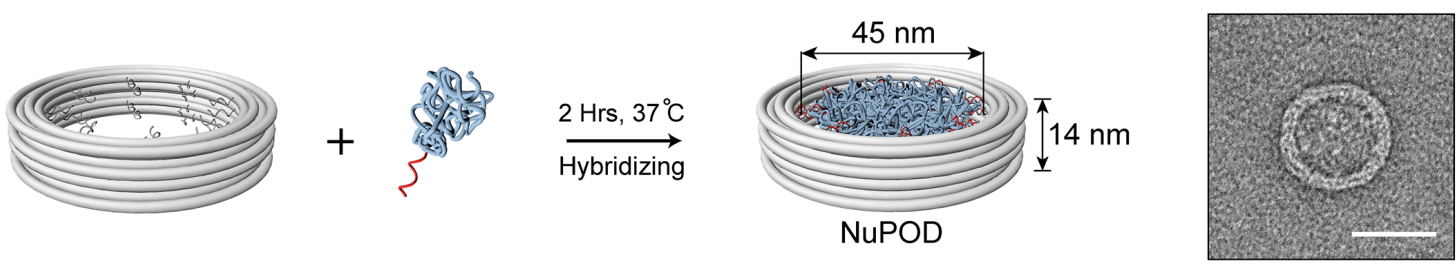

C
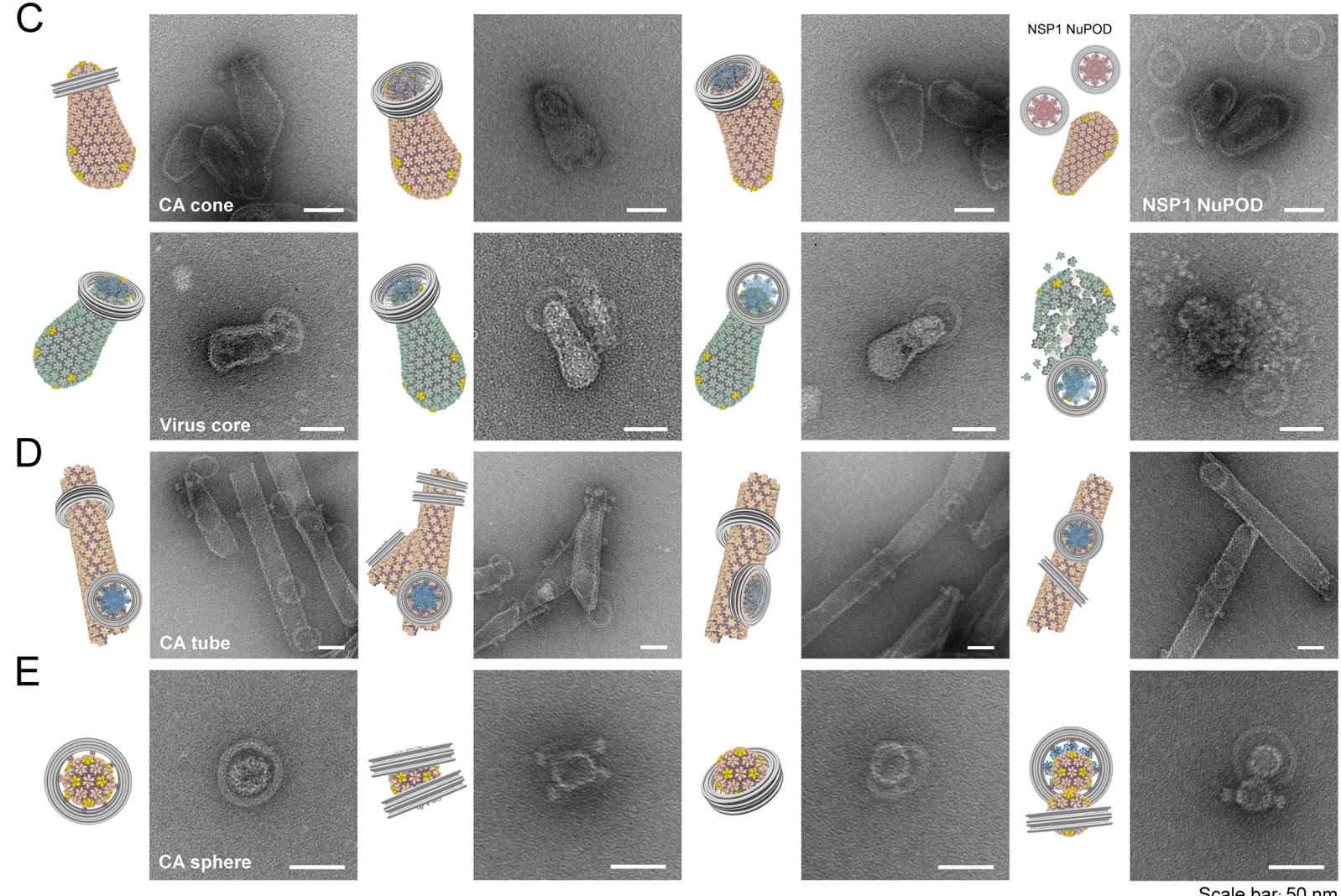

Figure 3. NUP153 NuPOD binding to capsid assemblies.

(A) Schematic of conjugating anti-handle (BG-DNA oligonucleotide) to NUP153-SNAP (left). Right: SDS-PAGE analysis of the conjugation product compared to the original unconjugated protein. Note the shift in band positions.

(B) Construction of NuPOD to mimic critical features of the NPC. Left: schematic of NUP153SNAP-oligo hybridization to a DNA origami ring. Right: negative-stain electron micrograph of a 
NUP153CTD grafted NuPOD. Scale bars: $50 \mathrm{~nm}$.

(C) Schematics and negative-stain electron micrographs of the NUP153 NuPOD bound to in vitro assembled capsid cones (top) and purified native virus cores (bottom). Assembled capsid cones did not detectably bind to the yeast nucleoporin NSP1 NuPOD (top right). The NUP153 NuPOD also bound partially disassembled native cores (bottom right). Scale bars: $50 \mathrm{~nm}$.

(D) Schematics and negative-stain electron micrographs of the NUP153 NuPOD bound to CA tubes, either on side surfaces or with CA tubes threading through the NuPOD. Scale bars: $50 \mathrm{~nm}$.

(E) Schematics and negative-stain electron micrographs of the NUP153 NuPOD bound to CA spheres. Scale bars: $50 \mathrm{~nm}$.

See also Figure $S 4$.

We next tested the binding of native virus cores with the NUP153 NuPOD. Native cores were prepared from concentrated HIV-1 particles by sucrose density gradient centrifugation (Shah and Aiken, 2011). The results were similar to those obtained using in vitro assembled capsid cones; the tip regions of the native cores could bind and insert into the NUP153 NuPOD (Figure 3C). These findings suggest that NUP153 may have a curvature preference, specifically targeting the tips of HIV-1 capsid (described more below). Interestingly, the native core preparations contained partially dissolved cores, which likely contain deformed or partially disassembled capsids. We also observed NuPOD rings binding these partially disassembled structures. Because NUP153 targets higher-order tri-hexameric interfaces on the capsid (Figure 1 and 2), these partially dissolved cores likely retained this aspect of the lattice structure (Figure $3 \mathrm{C}$, bottom right).

\section{CA tubes bind and thread through the NUP153 NuPOD}

We also investigated the interaction of the NUP153 NuPOD with CA tubes. As above, A14C/E45C cross-linked CA tubes (diameter $40-50 \mathrm{~nm}$ ) were used due to their stability under low salt conditions (Lopez et al., 2011) (Figure S4). NUP153 NuPOD readily bound CA tubes, often on the flat side surfaces (Figure 3D). Strikingly, we also observed CA tubes completely penetrating the entire depth of and threading through the NuPOD rings, sometimes with more than one attached at the tube tip (Figure 3D). This result provides direct evidence of the ability of intact capsid assemblies to pass through the central channel of a nuclear pore-like confinement. The passage of CA tubes presumably occurred for those with a diameter less than that of the NuPOD, although our data cannot tell if there was local constriction caused by NUP153 at the site of contact. We observed that the NUP153 NuPOD rings "glide" far along CA tubes, indicating that NUP153 can diffuse along the CA lattice. It is not clear whether there is directionality to this diffusion.

\section{NUP153 prefers to bind regions of high capsid curvature}

The observed dominant tip-binding mode of capsid cones to NUP153 NuPODs prompted us to investigate whether NUP153 has a curvature preference for binding. The cone-shaped capsid has a "flat" surface on its sides and highly curved tip regions (Liu et al., 2015) (Figure 4A). It is well established that the CA tube is a good mimic of 
the flat side of the capsid (Zhao et al., 2011). Additionally, it was reported that the N21C/A22C CA mutant can assemble into CA spheres $(30-45 \mathrm{~nm})$ that mimic the highly curved capsid tips (Zhang et al., 2018; Zhao et al., 2011) (Figure 4A and S4). We assembled N21C/A22C CA spheres and confirmed that these bound well to the NUP153 NuPOD, mostly residing in the middle of the NuPOD rings. When the NuPODs were in excess, we observed two NuPOD rings attaching to the same CA sphere (Figure 3E).

A
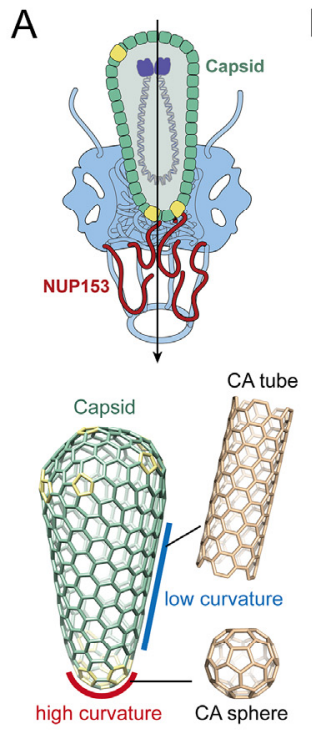

C

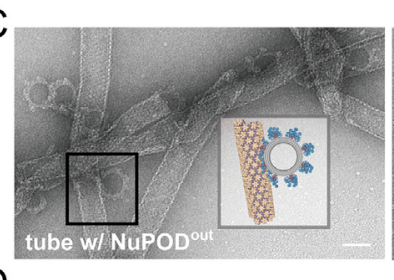

$\mathrm{D}$

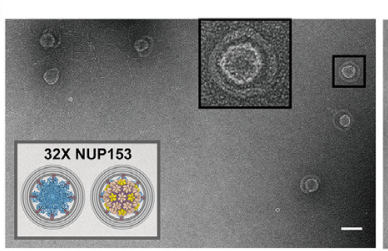

B
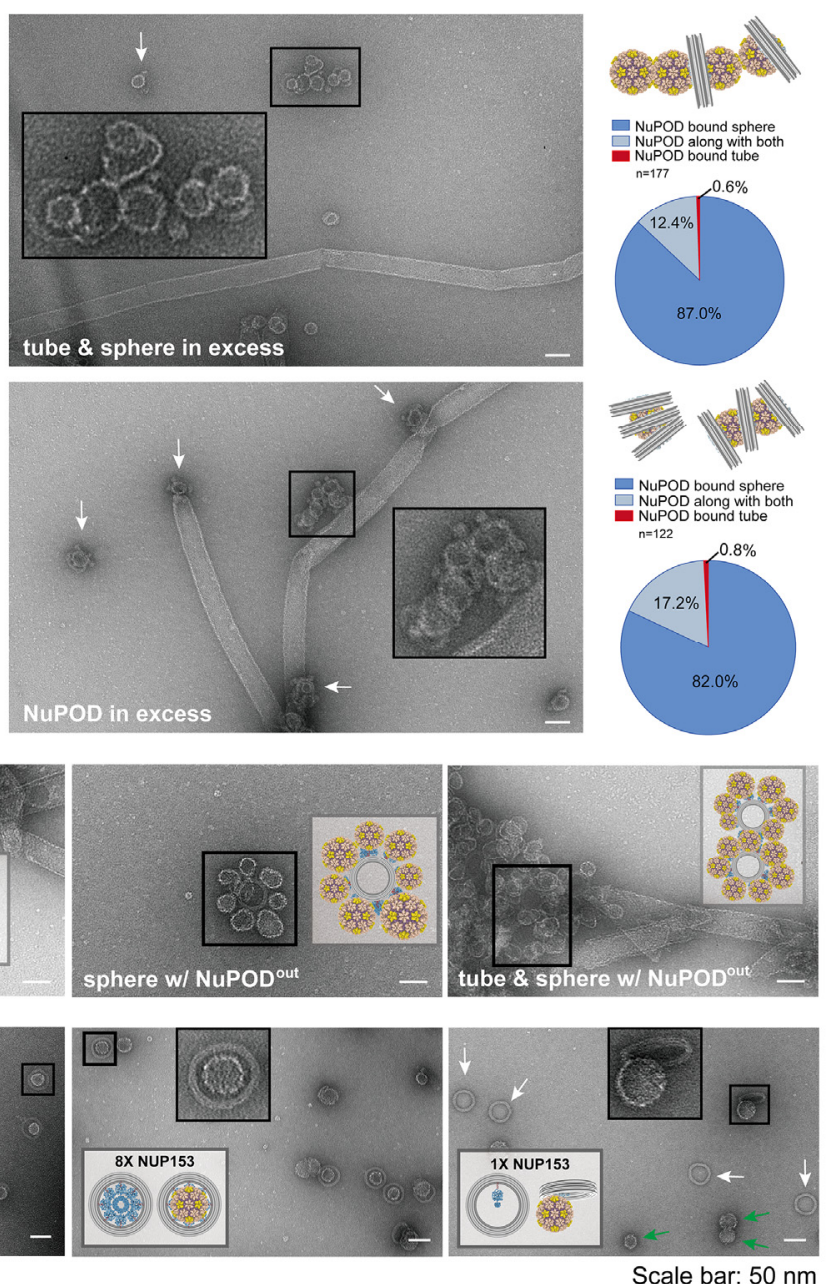

Figure 4. NUP153 prefers highly-curved regions of capsid for avid NuPOD association.

(A) Top: Schematic of capsid tip insertion into the NPC central pore during HIV-1 nuclear transport. Bottom: Schematics showing that the capsid cone has a flat surface on its sides and higher curvature at the tips. CA tubes mimic the sides of the capsid and CA spheres resemble the tips.

(B) Left: Negative-stain electron micrographs of NUP153 NuPOD binding to a 1:1 mixture of CA spheres and tubes in excess (top) versus binding when the NuPOD was in excess (bottom). The white arrows and insets mark the sphere-bound NuPODs. Right: Schematics of the major binding mode and quantification of all binding events. Quantifications suggest a sphere-preferred binding of NuPOD (Statistical significances were determined by the Chi-Squared Test, $\mathrm{P}<0.0001$ ). Scale bars: $50 \mathrm{~nm}$.

(C) Negative-stain electron micrographs of the external-grafted NUP153 ${ }^{\text {out }}$ NuPOD binding to CA 
tubes alone (left), CA spheres alone (middle), and a 1:1 mixture of CA spheres and tubes (right). Note the lack of CA sphere binding to the flat tube surface in the mixture experiment. Scale bars: $50 \mathrm{~nm}$.

(D) Negative-stain electron micrographs of CA spheres binding to NuPODs with 32 (left), 8 (middle), or 1 (right) copies of NUP153CTD. Most of the single-NUP153 NuPOD remain empty without bound CA spheres (right, marked with white arrows). The free spheres are marked with green arrows. Scale bars: $50 \mathrm{~nm}$.

CA tube and sphere assemblies capture two different surface geometries present in native capsids. We accordingly used these assemblies in binding competition assays to elucidate the curvature preference of NUP153. NUP153 NuPODs challenged with equimolar mixtures of CA spheres and tubes were analyzed by negative-stain EM (Figure 4B). We first applied a tube:sphere mixture (1:1 ratio) in excess of the NuPODs, and observed that almost all NuPODs bound to CA spheres, including some NuPODs with two spheres attached. Quantification revealed that close to $90 \%$ of the NuPODs bound CA spheres only, compared to 0.5\% bound to CA tubes, which was over a 100-fold difference (Figure 4B, bottom left). When NuPODs were added in excess of the CA sphere:tube mixture (1:1 ratio), we again found over a 100-fold preference for sphere binding over tubes (Figure 4B, bottom right). The excess NuPODs did not lead to increased binding to $C A$ tubes, but rather greater NuPOD occupancy (up to three) bound to the same CA sphere. As the CA spheres mimic the capsid tip geometry, this is consistent with our observation that the NUP153 NuPOD preferentially bound to the tips of either in vitro assembled capsid cones or purified virus cores (Figure $3 \mathrm{C}$ ).

We note that because NUP153 was positioned inside the NuPOD ring, the observed CA sphere preference can be potentially due to geometric constraints leading to greater inaccessibility of CA tubes. To exclude this possibility, we repositioned the DNA handles to the exterior of the origami ring to create a NuPOD with fully exposed NUP153CTD. This exterior-grafted NUP153 NuPOD (NUP153 ${ }^{\text {out }}$ NuPOD) efficiently bound CA tubes (on the flat side) and spheres (6-8 spheres per NuPOD) in tube-only or sphere-only reactions (Figure $4 C$ ). Consistent with the earlier competition results, the majority of NUP153 ${ }^{\text {out }}$ NuPODs clustered around the CA spheres in the mixture experiment, without binding to the flat surface of CA tubes (Figure 4C). These results confirm that the observed preference for capsid-tip curvature is an intrinsic property of NUP153. It is conceivable that this preference facilitates the directional transport of the capsid through the NPC.

\section{Multiple copies of NUP153 provide binding avidity to the capsid}

As there are 32 copies of NUP153 in a natural NPC, we constructed NuPODs with varying numbers of NUP153CTD attached $(1,8$, or 32$)$ to understand how binding avidity affects the association of the capsid to the NPC (Figure 4D). NuPODs with either 8 or 32 NUP153 copies adhered robustly to CA spheres. By contrast, NuPODs with only 1 copy of NUP153 bound CA spheres poorly, with only $10 \%$ of NuPODs having 
attached spheres (Figure 4D). These results suggest that a single NUP153 molecule is insufficient for stable capsid attachment to the NPC and that binding avidity from multiple copies of NUP153 is needed to ensure strong association. Furthermore, this is consistent with the dynamic, rapidly transitioning bound and unbound states of NUP153 found in our MD simulations (Figure 2E). These fast on/off kinetics are potentially important for the translocation of the capsid within NPC, as suggested by CA tube threading through the NuPODs (Figure 3D).

A

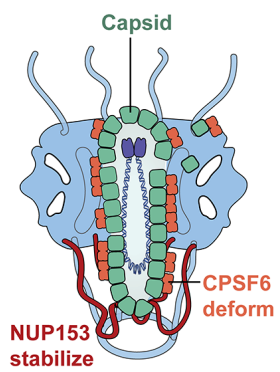

C CPSF6 deformed CA lattice

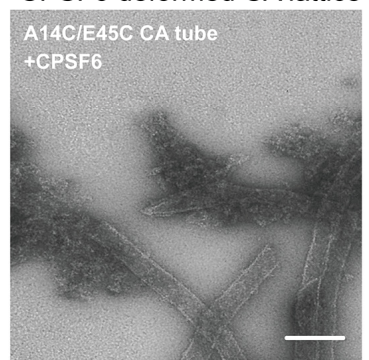

$\mathrm{B}$
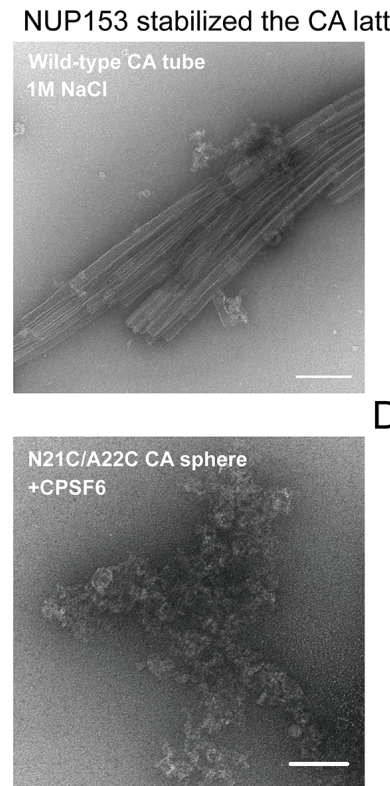

D
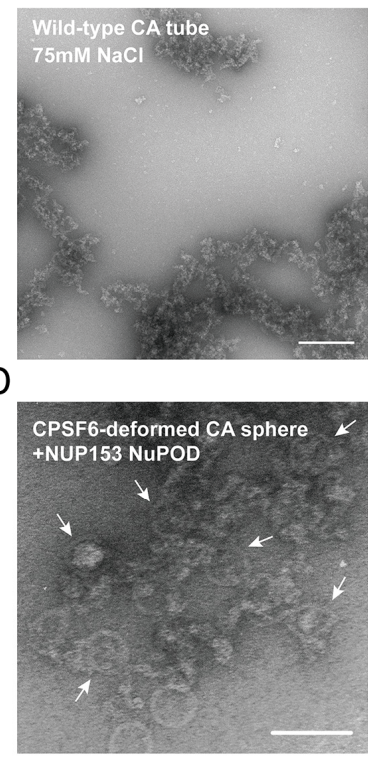
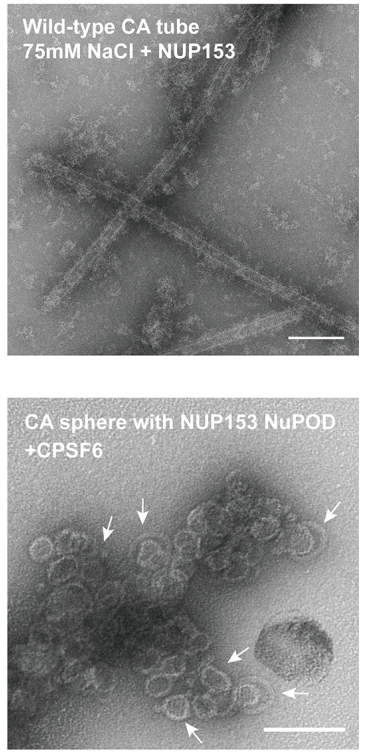

Figure 5. NUP153 stabilizes and CPSF6 destabilizes CA lattice.

(A) Schematic of the combined effect of NUP153 and CPSF6 on the capsid.

(B) Negative-stain electron micrographs of intact wt CA tubes under high salt conditions (1 M $\mathrm{NaCl}$, left), disintegrated wt $\mathrm{CA}$ tubes under low salt conditions (75 $\mathrm{mM} \mathrm{NaCl}$, middle), and wt CA tubes stabilized by NUP153 under low salt conditions ( $75 \mathrm{mM} \mathrm{NaCl}$, right). Scale bars: $200 \mathrm{~nm}$. (C) CFSF6 induces crosslinked CA tubes and spheres to disassemble and/or deform. Scale bars: $100 \mathrm{~nm}$.

(D) Left: NUP153 NuPOD attached to CPSF6-deformed CA spheres. Right: CFSF6 was added to a pre-incubated mixture of NUP153 NuPOD with CA spheres, which remained largely intact. Scale bars: $100 \mathrm{~nm}$.

See also Figure S5.

\section{NUP153 stabilizes capsid assemblies}

How and when the capsid uncoats during HIV-1 infection remains a longstanding question (Hofer, 2020). To better understand the potential involvement of NUP153 in capsid uncoating, we tested its effect on wild-type (wt) CA tubes under different salt conditions. The wt CA tube was stable in a high salt environment such as $1 \mathrm{M} \mathrm{NaCl}$, but its lattice structure rapidly collapsed and disintegrated when the salt concentration was lowered to $75 \mathrm{mM}$ (Figure 5B). However, tube disassembly at low salt concentration was averted in the presence of NUP153 $3^{1411-1475}$, resulting in a large number of intact tubes coated with fuzzy irregularly-shaped NUP153 ${ }^{1411-1475}$ proteins 
(Figure 5B). Similar results were obtained when testing the effect of NUP153 $1411-1475$ on CA spheres using size-exclusion chromatography assays. NUP153 stabilized CA spheres under low salt conditions, preventing their disassembly (Figure S5). The stability effect of NUP153 ${ }^{1411-1475}$ was concentration dependent. A large number of micron-long CA tubes could be observed at $50 \mu \mathrm{M}$ NUP153, some tubes of nanometers in length remained at $5 \mu \mathrm{M}$ NUP153, and a complete loss of tube structures resulted at $1 \mu$ M NUP153 (Figure S5). Given its high local concentration in the NPC, NUP153 binding to the capsid likely does not trigger uncoating but rather helps stabilize the lattice.

\section{CPSF6 destabilizes capsid assemblies}

One of the most intriguing aspects of HIV-1 nuclear import is the apparent dimensional mismatch between the $\sim 60 \mathrm{~nm}$ wide capsid and the $\sim 40 \mathrm{~nm}$ diameter of the nuclear pore, although heterogeneity in NPC central pore sizes has been suggested (Beck and Baumeister, 2016; Feldherr et al., 2001; Mahamid et al., 2016). This problem can potentially be resolved by the involvement of other host factors that can help remodel the capsid structure. CPSF6 facilitates capsid nuclear import and may play such a role in this process. It has been reported that truncated CPSF6 deforms and disrupts CA tubes (Ning et al., 2018). Indeed, we found that full-length CPSF6 $(1 \mu \mathrm{M})$ efficiently disrupted CA spheres and tubes into potentially deformed CA lattices (Figure 5C and S5). NUP153 NuPODs could still bind these largely deformed spheres (Figure 5D, left), which is reminiscent of our observation of NuPOD binding to deformed native virus cores (Figure $3 C$, bottom right). When CA spheres were pre-incubated with the stabilizing NUP153 NuPODs, the destructive effect of CPSF6 was significantly reduced (Figure 5D, right). It is possible that the combined effect of these two host factors allows the capsid to deform yet remain largely intact to pass through NPCs.

\section{Discussion}

HIV-1 nuclear import is one of the least understood processes of the viral life cycle. It was initially thought that the capsid immediately uncoated upon virus entry into the cell, with only recent studies pointing to the possibility of a largely intact capsid passing through the NPC into the nucleus (Burdick et al., 2020). However, mechanistic data regarding this process remain scarce. In this study we provide indepth mechanistic insights into how HIV-1 capsid interacts with a critical NPC component, NUP153, revealing the likely import mechanism. We show that besides the known FG-motif that binds weakly to CA hexamers, NUP153 contains a previously unexplored 'RRR' motif at its C-terminus that is predominantly responsible for its binding to the HIV-1 capsid. Together, these two regions enable NUP153 to strongly interact with the capsid (Figure 2C). We also created a minimal 25 aa peptide composed of the FG and RRR-motifs that retained potent capsid binding in vitro and in the context of HIV-1 infection.

The NUP153 C-terminal region senses a pattern in capsid formed via the tri- 
hexamer center that only exists in the assembled capsid. This binding characteristic has profound implications on the mode by which the capsid engages the NPC to enter the nucleus. Although NUP153 was found to be able to extend to the cytosol (Fahrenkrog et al., 2002), it is part of the NPC nuclear basket likely positioned deep into the nucleus. The higher-order CA lattice targeting mode of NUP153 supports nuclear penetration of the capsid that is at least partially assembled, especially if the brunt of the many copies of NPC-associated NUP153 engage the capsid during nuclear import. These findings establish a mechanistic foundation for passage of the intact or nearly intact capsid into the nucleus (Burdick et al., 2020).

We have designed and employed NuPODs, a unique and modular experimental tool for gaining mechanistic insights into nuclear transport mechanisms. Our NuPOD system allowed us to interrogate previously unobtainable mechanistic details of HIV1 capsid nuclear entry. For example, the NUP153 NuPOD showed an unambiguous preference to bind the tip regions of the capsid. This high-curvature preference of NUP153 potentially facilitates the correct alignment of the end of the capsid to the NPC for penetration. Rather strikingly, we captured CA tubes threading through NUP153 NuPOD rings, which may represent the scenario of capsid passing through the NPC when the central pore is of sufficient size (Figure 6). Although it is thought that the diameter of the NPC channel is less than the width of an HIV-1 capsid, recent evidence has shown that the NPC possesses a degree of elasticity, and the central channel may expand under certain conditions (Mahamid et al., 2016; Zhang et al., 2020). Consistently, our data provide direct evidence that assembled capsid structures can pass through a nuclear pore-like channel (Figure 3D).

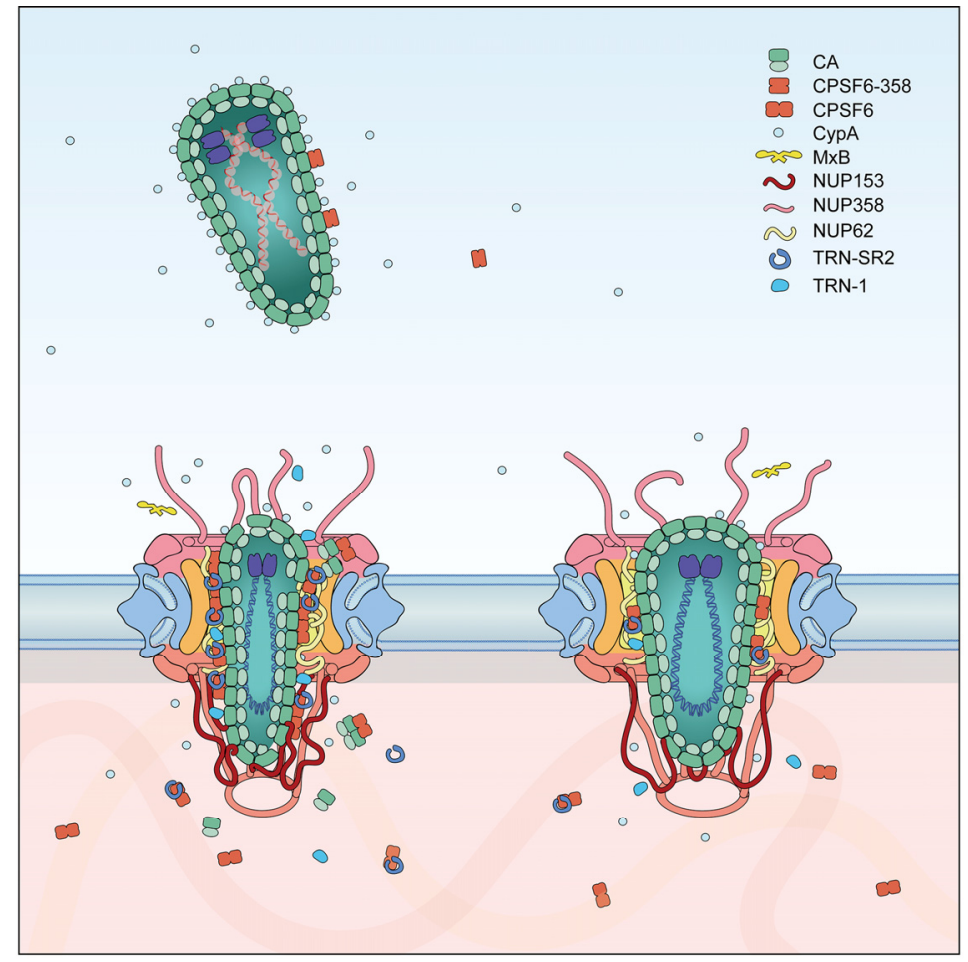

Figure 6. Model of the capsid passing through small (left) and large (right) NPC. 
In addition to NUPs, other proteins, such as CPSF6, interact with the HIV-1 capsid to facilitate its nuclear import (Bejarano et al., 2019; Chin et al., 2015). These proteins may modulate capsid stability in opposite directions to achieve a fine balance to allow its passage through the NPC. The CPSF6-capsid interaction has also been implicated in post-entry trafficking of capsids to the interior region of the nucleus for integration (Achuthan et al., 2018; Burdick et al., 2020; Francis et al., 2020). CPSF6 deforms and causes the disintegration of capsid assemblies, as shown in our experiments and reported previously for a CPSF6 truncation variant (Ning et al., 2018). We found that NUP153 can counteract the destabilization effect of CPSF6 (Figure 5B, D). The combined effect of these two factors may enable the capsid to deform and morph through NPCs of comparatively small size and still enter into the nucleus largely intact (Figure 5D and 6). Recent studies have indicated that the capsid could remain at the nuclear pore for a few hours during its transit through the NPC (Burdick et al., 2020; Zuliani-Alvarez and Towers, 2019). One can picture a scenario whereby multiple copies of NUP153 in the NPC bind the narrow tip of the capsid to keep it positioned while CPSF6 exerts local deformations to enable the capsid structure to pass through the pore (Figure 6).

In the context of viral infection, after capsid entry into the host cell, the outcome of the infection depends on a complex network of interactions between host and viral proteins. For example, cytoplasmic CypA may attach to the capsid to protect the virus against the host immune response (Kim et al., 2019; Rasaiyaah et al., 2013), and it may also compete with NUP358 and affect capsid docking to the NPC. MxB in the vicinity of the nucleus may bind to the capsid lattice and restrict its entry into the NPC. Within the NPC and inside the nucleus, cooperation and competition between CPSF6 and other proteins such as CypA (Schaller et al., 2011) and NUP153 (Di Nunzio et al., 2012; Koh et al., 2013) may occur to enable the delivery of the capsid to sites of integration. Considering the versatility of these proteins and their interactions with each other, the network of proteins involved in the nuclear transport process is complex and intertwined (Figure 6). Perturbations in one or a subset of these proteins may lead to convoluted effects on the progress of the capsid through the NPC. This complexity presents a major hurdle for mechanistic studies of nuclear transport in cells. The NuPOD platform is a unique toolkit to enable a biochemical approach to one of the most complex aspects of cell biology. Herein, we leveraged a simple NuPOD system to gain mechanistic insight into the nuclear passage of HIV-1. With modularity and programmability, next-generation NuPODs can be built with increasing complexity to more closely mimic the environment of NPC. We envision NuPODs will continue to be an invaluable tool to study HIV-1 nuclear import as well as other viruses that rely on their capsids to gain nuclear entry (Fay and Pante, 2015; Gallucci and Kann, 2017). 


\section{Methods}

\section{EXPERIMENTAL MODEL AND SUBJECT DETAILS}

Molecular cloning, as well as expression of recombinant proteins, was carried out in competent $E$. coli strain BL21 (DE3). Cells were cultured at $37^{\circ} \mathrm{C}$, in either Luria Broth (LB; for starter culture) or Terrific Broth (TB; for protein expression), while shaking at 220 RPM. HEK293T were cultured in Dulbecco's modified Eagle medium supplemented to contain $10 \%$ fetal bovine serum (FBS), $100 \mathrm{lU} / \mathrm{ml}$ penicillin, and $0.1 \mathrm{mg} / \mathrm{ml}$ streptomycin at $37^{\circ} \mathrm{C}$ with $5 \% \mathrm{CO}^{2}$ in a humidified incubator. HIV-1 particles were harvested from MT-4 cells infected with HIV-1 molecular clone R9. MT-4 cells were cultured in RPMI1640 medium supplemented with fetal bovine serum (10\% vol/vol), penicillin (100 units $/ \mathrm{ml})$, and streptomycin $(0.1 \mathrm{mg} / \mathrm{ml})$ in a humidified incubator at $37^{\circ} \mathrm{C}$ and $5 \% \mathrm{CO}^{2}$.

\section{METHOD DETAILS}

\section{Cloning and expression}

NUP153CTD from homo sapiens (amino acids 896-1475), as well as yeast nucleoporin NSP1 (amino acids 2-603) from S. cerevisiae, was cloned as 10x His-MBP-SUMONUP153 (or NSP1)-SNAP constructs (Figure S1) into a pET-28a-derived vector (Novagen) and expressed in BL21-Gold (DE3). Cells were grown in TB at $37^{\circ} \mathrm{C}$ with shaking until they reached an $\mathrm{OD}_{600}$ of 0.8 . Protein expression was then induced with $1 \mathrm{mM}$ IPTG for $3 \mathrm{hrs}$ at $20^{\circ} \mathrm{C}$ before collection by centrifugation. Cell pellets were refrigerated at $-80^{\circ} \mathrm{C}$ until use.

All CA constructs were cloned into pET-11a (EMD Millipore). CA-Foldon was generated by directly fusing Foldon to the C-terminus of CA. CA proteins were overexpressed in BL21-Gold (DE3) cells at $25{ }^{\circ} \mathrm{C}$ for $12 \mathrm{hr}$ by induction with $0.5 \mathrm{mM}$ IPTG at $0.8 \mathrm{OD}_{600}$. Cell pellets were collected by centrifugation and refrigerated at $-80{ }^{\circ} \mathrm{C}$ until use. Human CPSF6 was cloned into bacterial expression vector and expressed.

\section{Protein purification}

NUP153/NSP1-expressing cell pellets were resuspended in lysis buffer (1× PBS, 0.5 $\mathrm{mM}$ TCEP, $0.1 \mathrm{mM}$ PMSF, $1 \times$ Roche cOmplete protease inhibitors, $\mathrm{pH} 7.4$ ), and lysed in a homogenizer. Whole-cell lysates were then spun at 35,000 rpm for $45 \mathrm{~min}$ in a Type 45 Ti rotor (Beckman Coulter). Subsequently, the supernatant was decanted and filtered through a $0.45 \mu \mathrm{m}$ cellulose acetate membrane. The resulting filtered lysate was spiked with $25 \mathrm{mM}$ imidazole, then applied to a $5 \mathrm{~mL}$ HisTrap column (GE) on an ÄKTA system (GE) at a flow rate of $1 \mathrm{~mL} / \mathrm{min}$. The column was rinsed with a wash buffer (1× PBS, $25 \mathrm{mM}$ imidazole) and eluted on a buffer gradient (1×PBS, $500 \mathrm{mM}$ imidazole). Concentrations of purified protein were determined by Nanodrop (Thermo Fisher). Samples were flash-frozen in liquid nitrogen and refrigerated at $-80^{\circ} \mathrm{C}$ until use. 
Untagged CA proteins were purified by $25 \% \mathrm{w} / \mathrm{v}$ ammonium sulfate precipitation, dialysis into a low-salt buffer ( $25 \mathrm{mM}$ HEPES, $0.1 \mathrm{mM}$ TCEP, $\mathrm{pH}$ 7), and cation exchange chromatography. CA-foldon fusions were purified by $35 \% \mathrm{w} / \mathrm{v}$ ammonium sulfate precipitation and anion exchange chromatography. All CA constructs were dialyzed into CA storage buffer before freezing or further experiments $(50 \mathrm{mM}$ Tris, $75 \mathrm{mM} \mathrm{NaCl}$, $40 \mathrm{mM}$ BME, $\mathrm{pH}$ 8). All purification steps were validated using SDS-PAGE.

\section{CA tube co-pelleting assays}

A14C/E45C disulfide crosslinked CA tubes were dialyzed into $25 \mathrm{mM}$ Tris, $\mathrm{pH} 8$ buffer. NUPs were incubated in $21 \mathrm{uL}$ reactions with CA tubes for $30 \mathrm{~min}$ at room temperature, then spun at $20000 \mathrm{~g}$ for $10 \mathrm{~min}$ at $4{ }^{\circ} \mathrm{C}$. Total, soluble, and pellet fractions were collected and analyzed using SDS-PAGE. NUP153CTD and all mutant constructs were incubated at $3 \mu \mathrm{M}$ with $100 \mu \mathrm{M}$ CA in a $25 \mathrm{mM}$ Tris, $75 / 300 \mathrm{mM} \mathrm{NaCl}, \mathrm{pH} 8$ buffer.

\section{Size-exclusion chromatography co-elution assays}

Assembled CA hexamer, hexamer-2, and pentamer were mixed with NUP153 ${ }^{1411-1475}$ for $30 \mathrm{~min}$ to $1 \mathrm{hr}$ on ice in the same buffer used for CA tube co-pelleting assays (unless specified). Binding reactions were carried out in a volume of $100 \mathrm{uL}$ and contained 12 $\mu \mathrm{M}$ NUPs and $72 \mathrm{uM}$ monomeric concentration of CA. All binding tests were performed on GE Superdex $20010 / 300 \mathrm{GL}$ column in a $25 \mathrm{mM}$ Tris, $75 \mathrm{mM} \mathrm{NaCl}, \mathrm{pH} 8$ buffer with a flow rate of $0.5 \mathrm{ml} / \mathrm{min}$. The $280 \mathrm{~nm}$ absorbance was recorded.

Size-exclusion chromatography for assembled N21C/A22C CA sphere, NUP153 $3^{1411-1475}$, and mixtures were performed on GE Superose $610 / 300 \mathrm{GL}$ column in $25 \mathrm{mM}$ Tris buffer $\mathrm{pH} 8,50 / 1000 \mathrm{mM} \mathrm{NaCl}$. The $280 \mathrm{~nm}$ absorbance was recorded.

\section{Isothermal titration calorimetry}

Binding reactions were performed in a TA Instruments NanolTC machine at $25^{\circ} \mathrm{C}$ in a $25 \mathrm{mM}$ Tris, $75 \mathrm{mM} \mathrm{NaCl}, \mathrm{pH} 8$ buffer. Hexamer-2 was stable in these conditions after overnight dialysis and during experiments. Synthesized NUP153 peptide (Genscript Co.) was resuspended in the same buffer and injected as the titrant to the Hexamer-2 in the cell. Data were analyzed using the NanoAnalyze (TA Instruments) software, and curves were fitted with an independent one-site binding model.

\section{Size exclusion chromatography linked to multi-angle light scattering (SEC-MALS)}

Multiangle laser light-scattering experiments were performed at room temperature in a $50 \mathrm{mM}$ Tris- $\mathrm{HCl}, 150 \mathrm{mM} \mathrm{NaCl}, \mathrm{pH} 8$ buffer. Light-scattering data were measured using a Dawn Heleos-II spectrometer (Wyatt Technology) coupled to an Opti-lab TrEX(Wyatt Technologies) interferometric refractometer. Samples (100 uL) at $1 \mathrm{mg} / \mathrm{mL}$ were injected and run over a Superdex $200 \mathrm{GL}$ (GE Healthcare) column at a $0.5 \mathrm{ml} / \mathrm{min}$ flow rate. Data on light scattering ( $690 \mathrm{~nm}$ laser), $280 \mathrm{~nm}$ absorbance, and refractive index were measured. Before sample runs, the system was calibrated and normalized using the isotropic protein standard, monomeric bovine serum albumin. The $\mathrm{dn} / \mathrm{dc}$ 
value (changes in solution refractive index with respect to protein concentration) is relatively constant for proteins (Wen, 1996), and set to 0.185 for all experiments and analysis. Data were processed in ASTRA as previously described (Wyatt, 1993).

\section{Trim-NUP153 mediated restriction assays}

\section{Plasmid construction}

TRIM-NUP153 proteins were expressed from the bicistronic expression vector pIRES2-eGFP (Shun et al., 2007). To make Trim-HA- NUP153896-1475, Trim and NUP153 sequences were PCR-amplified from pLPCX-Trim-HA-NUP153c (Matreyek et al., 2013) using primer pairs 5'-GATCTCGAGCTCAAGCTTCGAATTC/5'GTAATCTGGAACATCGTATGGGTAGCCACCTCCAGATCCCCAGTAGCGTCGG3' and 5'CATACGATGTTCCAGATTACGCTGGAGGTGGATCTCCGCGGAACTCAGCAGCCTCCTC $/ 5^{\prime}$ GATCCCGGGCCTTTATTTCCTGCGTCTAACAG, respectively (Sac // restriction site underlined). The linked TRIM-HA-NUP153 insert, amplified from these DNAs using primers 5'-GATCTCGAGCTCAAGCTTCGAATTC and 5'-

GATCCCGGGCCTTTATTTCCTGCGTCTAACAG, was digested with $X$ ho $I$ and $X m a$ I and ligated with Xho I/Xma I-digested pIRES2-eGFP. Trim-HA-NUP153 ${ }^{1401-1475}$ vector was created by amplifying NUP153 $3^{1401-1475}$ sequences using primers $5^{\prime}$ CAGCCGCGGACTACAAATTTCAACTTCACAAACAACAG and 5'GATCCCGGGCCTTTATTTCCTGCGTCTAACAG, followed by digestion with Sac II and Xma I and swapping this DNA for the analogous region within Trim-HA-NUP153 ${ }^{896-1475}$ via Sac II and Xma / restriction sites. To create Trim-HA-NUP153 ${ }^{1411-1475}$ lacking residues of 1425-1464, double-stranded DNA representing NUP153 residues 1411-1424 and 1465-1475 was synthesized by IDT (5'CAGCCGCGGCCATCAGGAGTGTTCACATTTGGTGCAAATTCTAGCACACCTCGCAAGATAA AGACTGCTGTTAGACGCAGGAAATAAAGGCCCGGGATC3'), digested with Sac // and Xma I, and swapped for the NUP153 ${ }^{896-1475}$ region as above. TRIM-NUP coding regions of all plasmids were verified by DNA sequencing.

\section{Cells, virus production, and infection}

HEK293T cells were cultured in Dulbecco's modified Eagle medium supplemented to contain $10 \%$ fetal bovine serum (FBS), $100 \mathrm{lU} / \mathrm{ml}$ penicillin, and $0.1 \mathrm{mg} / \mathrm{ml}$ streptomycin.

Single-round HIV-1 carrying the gene for firefly luciferase (HIV-Luc), produced by transfecting HEK293T cells in $10 \mathrm{~cm}$ dishes with $7.5 \mu \mathrm{g}$ pNLX.Luc.R-. $\Delta$ Avrll and $1.5 \mu \mathrm{g}$ pCG-VSV-G using PolyJet (SignaGen Laboratories), was concentrated by ultracentrifugation as described (Serrao et al., 2016). HIV-Luc yield was assessed by p24 ELISA (Advanced Bioscience Laboratories). Cells transfected with pIRES2-eGFP expression constructs were used in HIV infection assays as previously described (Jang et al., 2019). Briefly, HEK293T cells in 6-well plates were transfected with $2 \mu \mathrm{g}$ pIRES2eGFP or Trim-HA-NUP153 derivatives using Effectene (Qiagen). At $24 \mathrm{~h}$ posttransfection, GFP-positive cells were selected in basic sorting buffer ( 1 mM EDTA, 25 $\mathrm{mM}$ HEPES, $\mathrm{pH}$ 8.0, 1\% FBS, Mg2+/Ca2+-free phosphate-buffered saline) by 
fluorescence-activated cell sorting. Approximately $3-5 \times 10^{5}$ sorted cells were lysed for immunoblotting while $\sim 2 \times 10^{5}$ cells were infected in duplicate with HIV-Luc $(0.25 \mathrm{pg}$ p24 per cell) in the presence of $4 \mu \mathrm{g} / \mathrm{ml}$ polybrene. At $48 \mathrm{~h}$ post-infection, cells were lysed and luciferase activity was determined as previously described (Lu et al., 2004). Luciferase values were normalized to the level of total protein in cell lysates as determined by the BCA assay (Pierce).

\section{Western Blotting}

Details of the procedure were as described (Jang et al., 2019). $10 \mu \mathrm{g}$ of total cellular proteins fractionated through $3-8 \%$ polyacrylamide Tris-acetate or $4-12 \%$ polyacrylamide Bis-Tris gels (Invitrogen) were subsequently transferred to polyvinylidene difluoride (PVDF) membranes. Fusion protein expression was detected with anti-hemagglutinin (HA) antibody sc7392 (Santa Cruz) followed by HRP-mouse IgG antibody (Dako); actin was detected by HRP-conjugated $\beta$-actin antibody (Abcam). Membranes developed using ECL prime reagent (Amersham Biosciences) were imaged with a ChemiDoc MP imager (Bio-Rad).

\section{Molecular dynamics (MD) simulation}

Atomic model for MD simulation

The 3-dimension structure of the last 75 residues of NUP153 ${ }^{1401-1475}$ was built in Modeller (Eswar et al., 2006). A total number of 5,000 structures (Figure S3A) were generated and scored by the discreet optimized protein energy (DOPE) method (Shen and Sali, 2006). The candidate model of NUP153 ${ }^{1451-1475}$ was from the structure of the NUP153 ${ }^{1401-1475}$ with the lowest DOPE score and then subjected to a 100 -ns long equilibration in explicit water in NAMD2.13 (Phillips et al., 2005). The equilibrated NUP153 ${ }^{1451-1475}$ peptide was initially placed $1.5 \mathrm{~nm}$ above the tri-hexamer interface in the HIV-1 CA hexamer-2 model, which was built based on the structure of the full length native HIV-1 CA monomer (PDB:4XFX). The combined structure (Figure S3) was then solvated with TIP3P water (Jorgensen and Jenson, 1998) and neutralized by $\mathrm{NaCl}$ at $150 \mathrm{mM}$ concentration. The resulting model had a total number of 168,500 atoms (Figure S3B).

\section{MD simulation set-up}

After model building, the NUP153 ${ }^{1451-1475}$-CA hexamer-2 complex was subjected to a minimization and thermalization step. Subsequently, the whole system was equilibrated for over $100 \mathrm{~ns}$ at $310 \mathrm{~K}$. During the equilibration, the $\mathrm{C} \alpha$ atoms of the peripheral helices of CA monomer and the N-terminal residue in NUP153 ${ }^{1451-1475}$ were restrained. The minimization, thermalization and equilibration steps were completed in NAMD2.13 (Phillips et al., 2005). This equilibrated model then ran for a total simulation time of $8.7 \mu$ s on a special purpose computer Anton2 (Shaw et al., 2014) in the Pittsburgh supercomputing center. To mimic the CA hexamer lattice environment in the simulation, the $C \alpha$ atoms of three sets of CA $\alpha$-helices on the peripheries of the tri-hexamer model were applied with a harmonic restraint of $1 \mathrm{Kcal} / \mathrm{mol} \AA^{2}$ in $\mathrm{x}, \mathrm{y}$ and $z$ directions (Figure S3B). Also, the C $\alpha$ atoms of NUP153 residue 1451 was applied with 
a harmonic restraint of $1 \mathrm{Kcal} / \mathrm{mol} \AA^{2}$ in z direction. CHARMM $36 \mathrm{~m}$ (Huang et al., 2017) force field was used for all MD simulations. During the simulation, the temperature $(310 \mathrm{~K})$ and pressure (1 atm) was maintained by employing the Multigrator integrator (Lippert et al., 2013) and the simulation time step was set to $2.5 \mathrm{fs} / \mathrm{step}$, with shortrange forces evaluated at every time step, and long-range electrostatics evaluated at every second time step. Short-range non-bonded interactions were cut off at $17 \AA$; long range electrostatics were calculated using the k-Gaussian Split Ewald method (Shan et al., 2005).

\section{Markov state model analysis and Model validation}

Markov state models (MSMs) have been widely applied to study the conformational dynamics of biomolecules (Chodera and Noé, 2014; Husic and Pande, 2018b; Wang et al., 2018a). To identify the important metastable states of NUP153CTD in the MD simulation, Markov state models were constructed and validated in the PyEMMA 2.5.7 package (Scherer et al., 2015). First, the coordinates of NUP153 ${ }^{1451-1475}$ from the Anton2 simulation were directly transformed into two features, the Cartesian coordinates of NUP153 ${ }^{1451-1475}$ backbone atoms and 4,853 distance pairs between the backbone atoms of NUP153 $1451-1475$. Subsequently, time-lagged independent component analysis (TICA) (Pérez-Hernández et al., 2013) was performed to decompose these features onto 100 slow independent components (ICs) (Figure S3D). The projected data set was then discretized using the k-means method (MacQueen, 1967) and resulted in fifty microstates. The transition probability matrix between these microstates was then computed at a selected lag time of 1.2 ns (Figure S3J). Finally, an eight-state MSM using the PCCA+ algorithm (Röblitz and Weber, 2013) was constructed and the mean first passage times (MFPTs) between different states were estimated (Figure S3I). The eight-state MSMs built were validated with the ChapmanKolmogorov test (Noe et al., 2009; Prinz et al., 2011).

\section{DNA origami assembly}

DNA origami structures were designed in caDNAno (caDNAno.org). ssDNA handles were extended from the $3^{\prime}$ end of staple strands at positions indicated in Figure 3B. The handle sequences were 5'-AAATTATCTACCACAACTCAC-3' for inner/outer handles. These structures were assembled using an M13mp18 bacteriophage-derived circular ssDNA strand (8064 nt) and oligonucleotides from Integrated DNA Technologies over $36 \mathrm{hr}$ on an $85-25^{\circ} \mathrm{C}$ annealing gradient. Structures were purified using rate-zonal centrifugation through a 15-45\% glycerol gradient in a $1 \times \mathrm{TE},(5 \mathrm{mM}$ Tris- $\mathrm{Cl}, 1 \mathrm{mM}$ EDTA), $16 \mathrm{mM} \mathrm{MgCl}_{2}$, pH 8.0 buffer in an SW 55 rotor (Beckman Coulter) at 50,000 rpm at $4^{\circ} \mathrm{C}$ for $1 \mathrm{hr}$, followed by fraction collection (Lin et al., 2013). Fractions containing purified DNA origami structures were buffer exchanged into a $1 \times \mathrm{TE}, 16$ $\mathrm{mM} \mathrm{MgCl} 2, \mathrm{pH} 8.0$ buffer and stored at $-20^{\circ} \mathrm{C}$.

\section{Benzylguanine (BG)-DNA preparation}

5'-labeled amino-DNA oligonucleotides (anti-handles; IDT) were resuspended in deionized $\mathrm{H}_{2} \mathrm{O}$ at $2 \mathrm{mM}$ concentration. BG-NHS was dissolved in DMSO at $20 \mathrm{mM}$. Anti- 
handles and BG-NHS were mixed in a 1:3 ratio in 67 mM HEPES, pH 8.5 buffer, and incubated at room temperature for 1 hour. BG-DNA was then purified from excess BG-NHS by ethanol precipitation. Dried BG-DNA pellets were stored at $-20^{\circ} \mathrm{C}$ until use.

\section{Protein-DNA conjugation}

BG-DNA pellets were resuspended in de-ionized $\mathrm{H}_{2} \mathrm{O}$ and mixed with purified NUP153CTD in 1× PBS buffer at a final concentration of $40 \mu \mathrm{M}$ BG-DNA and $20 \mu \mathrm{M}$ NUP153CTD (2:1). This reaction was incubated at $25^{\circ} \mathrm{C}$ for $2 \mathrm{hr}$. Excess DNA was removed from conjugated proteins using size exclusion chromatography on a Superdex 200 10/300 GL column (GE Healthcare) in a 25 mM Tris, 75 mM NaCl, pH 8 buffer. Conjugation efficiency was verified by SDS-PAGE using Coomassie or SYPRO Red stain (Thermo Fisher).

\section{Hybridization of NUP to DNA origami}

DNA-conjugated NUP153CTD was added to DNA origami rings at $1.5 \times$ excess over the number of origami handles (e.g. $5 \mathrm{nM}$ origami $\times 32$ handles $\times 1.5=240 \mathrm{nM}$ FGNUP153-DNA) in a $1 \times \mathrm{TE}, 16 \mathrm{mM} \mathrm{MgCl}_{2}, \mathrm{pH} 8.0$ buffer and incubated for $2 \mathrm{hr}$ at $37^{\circ} \mathrm{C}$. NUP153 NuPODs were purified by rate-zonal centrifugation, as described in "DNA Origami Assembly", through a 15-45\% glycerol gradient in the hybridization buffer. The purified product is called a NUP153 NuPOD (NucleoPorins Organized on DNA).

\section{CA assembly}

Hexamer-2, hexamer and pentamer

Hexamer-2 was assembled using a 1:1 molar ratio of the appropriate CA proteins (Summers et al., 2019), resulting in $10-40 \mathrm{mg} / \mathrm{mL}$ of total proteins. Mixtures were dialyzed overnight (using Thermo Slide-a-lyzer dialysis cassettes) in a $50 \mathrm{mM}$ Tris, $1 \mathrm{M}$ $\mathrm{NaCl}, \mathrm{pH} 8$ buffer. Mixtures were dialyzed for a second night in a $50 \mathrm{mM}$ Tris, $\mathrm{pH} 8$ buffer. Hexamer-2 assemblies eluted from an anion exchange column at approximately $250 \mathrm{mM} \mathrm{NaCl}$. Each assembly was purified using a Superdex 200 PG or Superdex 200 GL size-exclusion chromatography column (GE Healthcare) ran with a $50 \mathrm{mM}$ Tris, 300 $\mathrm{mM} \mathrm{NaCl}$, pH 8 buffer. Hexamer (A14C/E45C/W184A/M185A) and pentamer (N21C/A22C/W184A/M185A) assemblies were dialyzed (using Thermo Slide-a-lyzer dialysis cassettes) for $48 \mathrm{hr}$ in a $50 \mathrm{mM}$ Tris, $\mathrm{pH} 8$ buffer without redox reagents. Then the crosslinked hexamers or pentamers were purified using a Superdex $200 \mathrm{GL}$ column. All assemblies were concentrated to $20-50 \mathrm{mg} / \mathrm{mL}$ and frozen at $-80{ }^{\circ} \mathrm{C}$ for storage.

\section{Crosslinked CA tube}

A14C/E45C disulfide crosslinked CA tubes were assembled by dialyzing (in Slide-ALyzer dialysis cassettes) CA A14C/E45C into a $50 \mathrm{mM}$ Tris, $1 \mathrm{M} \mathrm{NaCl}$, pH 8 buffer at 15 $\mathrm{mg} / \mathrm{mL}$ for one night, followed by dialysis into $50 \mathrm{mM}$ Tris for another night. The crosslinked tube was stored in a $50 \mathrm{mM}$ Tris, $50 \mathrm{mM} \mathrm{NaCl}$, pH 8 buffer and could remain stable for months. 


\section{Wild-type CA tube}

Wild-type CA tubes were assembled by dialyzing into a $50 \mathrm{mM}$ Tris, $1 \mathrm{M} \mathrm{NaCl}, \mathrm{pH} 8$ buffer at $5 \mathrm{mg} / \mathrm{mL}$ for three to four nights. Assemblies were stored in a $50 \mathrm{mM}$ Tris, 1 $\mathrm{M} \mathrm{NaCl}, \mathrm{pH} 8$ buffer.

\section{CA sphere}

Following a previously established protocol (Zhang et al., 2018), purified N21C/A22C CA proteins $(1 \mathrm{mg} / \mathrm{mL}$ ) were dialyzed into a $50 \mathrm{mM}$ Tris- $\mathrm{HCl}, 1 \mathrm{M} \mathrm{NaCl}, \mathrm{pH} 8.0$ buffer overnight at $4^{\circ} \mathrm{C}$.

\section{$\underline{\mathrm{CA} \text { cone }}$}

A14C/E45C CA protein was diluted in a $25 \mathrm{mM} \mathrm{MES}, 100 \mathrm{mM} \mathrm{NaCl}, \mathrm{pH} 6.0$ buffer to $200 \mu \mathrm{M}$, then mixed with a 20 to 40 fold molar excess of IP6 in solution. The assembly reaction was maintained at room temperature for 5 min until the appearance of cloudy CA assemblies. 200 uM CypA was then added to reduce the aggregation of the cones. The CA assemblies were diluted 20 times before observation under negative-stain TEM.

\section{Isolation of HIV-1 cores}

Native HIV-1 cores were prepared from concentrated HIV-1 particles by centrifugation through a layer of $0.5 \%$ Triton $\mathrm{X}-100$ into a linear sucrose density gradient as described (Shah and Aiken, 2011). For this purpose, HIV-1 particles were harvested from $200 \mathrm{ml}$ cultures of MT-4 cells (Pauwels et al., 1987) infected with undiluted virus harvested from 10-cm dishes transfected with the wild type HIV-1 molecular clone R9 (Gallay et al., 1997), which is nearly identical to NL4-3 in its protein coding sequences. To produce the virus, cells were cultured in RPMI1640 medium supplemented with fetal bovine serum $(10 \% \mathrm{vol} / \mathrm{vol})$, penicillin $(100$ units $/ \mathrm{ml})$, and streptomycin $(0.1 \mathrm{mg} / \mathrm{ml})$ in a humidified incubator at $37^{\circ} \mathrm{C}$ and $5 \% \mathrm{CO}^{2}$. For virus production in MT-4 cells, one hundred million cells were pelleted, resuspended in $50 \mathrm{ml}$ of fresh medium, and inoculated with a quantity of HIV-1 corresponding to $5000 \mathrm{ng}$ of p24 in the presence of DEAE-dextran $(20 \mathrm{mg} / \mathrm{ml})$ to promote infection. Cells were subsequently cultured for $24 \mathrm{~h}$, followed by removal of the inoculum by pelleting and aspiration. The cell pellet was resuspended in $200 \mathrm{ml}$ of fresh medium and cultured for 4 to 5 days. The extent of infection was assessed daily by monitoring the culture visually for cytopathic effects. At approximately 5 days after inoculation, the cells were pelleted by centrifugation and the supernatants collected and clarified by passing through a 0.45 $\mathrm{mM}$ pore-size vacuum filter. The HIV-1 particles were concentrated by ultracentrifugation, resuspended, and HIV-1 cores were isolated. The quantity of viral CA protein in the dense gradient fractions corresponding to HIV-1 cores was determined by p24 ELISA (Wehrly and Chesebro, 1997). The presence of intact HIV-1 cores in these fractions was ascertained by negative stain transmission electron microscopy using uranyl formate. Fractions containing HIV-1 cores were aliquoted and flash frozen in liquid nitrogen and stored at $-80^{\circ} \mathrm{C}$ until use. 


\section{Negative-stain electron microscopy}

Capsid assemblies with origami samples were deposited onto glow-discharged 400 mesh formvar/carbon-coated copper grids (Electron Microscopy Sciences). Grids were then stained with $2 \%$ uranyl formate. Imaging was performed on a JEOL JEM-1400 Plus microscope operated at $80 \mathrm{kV}$ with a bottom-mount $4 \mathrm{k} \times 3 \mathrm{k}$ CCD camera (Advanced Microscopy Technologies).

\section{Statistical analysis}

The data analysis was performed using the SPSS 26.0 software package (IBM, United States). All data were expressed in mean \pm standard deviation (SD) if not specifically addressed. For the co-pelleting assay, differences were tested by Student's t-test with variances verified by Fisher's F-test. NuPOD binding counting was analyzed by the ChiSquared goodness of fit test. $P<0.05$ were considered statistically significant.

\section{SUPPLEMENTAL INFORMATION}

\section{ACKNOWLEDGMENTS}

We thank the Xiong Lab for discussion. This work was supported by NIH grants P50AI150481 (Y.X., C.A., and A.N.E.), P30GM110758 (J.R.P.), R01AI052014 (A.N.E.), T32Al007386 (G.J.B.), R21GM109466 (C.L. and C.P.L.), R01GM105672 (C.P.L.), R01GM132114 (C.L.) and Collaboration Development Pilot Program awards from the Pittsburgh Center for HIV Protein Interactions (J.R.P. and C.L.), and a Singapore Agency for Science, Technology and Research Graduate Scholarship (Q.X.). Anton 2 computer time was provided by the Pittsburgh Supercomputing Center (PSC) through NIH Grant R01GM116961. The Anton 2 machine at PSC was generously made available by D.E. Shaw Research.

\section{AUTHOR CONTRIBUTIONS}

Conceptualization, Q.S., C.L., and Y.X.; Methodology, Q.S, C.X., S. J., C.A., J.R.P., A.N.E., C.P.L., C.L., Y.X.; Investigation; Q.S., C.X., S. J., Q.X., S.C.D., T.T., G.J.B., T.N.T., Y.H., S.Y., J.T., J.S., C.A., J.R.P., A.N.E., C.P.L., C.L., Y.X.; Writing -Original Draft, Q. S.; Writing Review \& Editing, Q.S, C.X., S. J., Q.X., S.C.D., J.T., C.A., J.R.P., A.N.E., C.P.L., C.L., and Y.X.; Funding Acquisition, C.A., J.R.P., G.J.B., A.N.E., C.P.L., C.L., and Y.X..

\section{DECLARATION OF INTERESTS}

The authors declare no competing interests.

\section{References}

Achuthan, V., Perreira, J.M., Sowd, G.A., Puray-Chavez, M., McDougall, W.M., Paulucci-Holthauzen, A., 
Wu, X.L., Fadel, H.J., Poeschla, E.M., Multani, A.S., et al. (2018). Capsid-CPSF6 Interaction Licenses Nuclear HIV-1 Trafficking to Sites of Viral DNA Integration. Cell Host \& Microbe 24, 392-+. Ao, Z.J., Jayappa, K.D., Wang, B.C., Zheng, Y.F., Kung, S., Rassart, E., Depping, R., Kohler, M., Cohen, E.A., and Yao, X.J. (2010). Importin alpha 3 Interacts with HIV-1 Integrase and Contributes to HIV-1 Nuclear Import and Replication. Journal of Virology 84, 8650-8663.

Beck, M., and Baumeister, W. (2016). Cryo-Electron Tomography: Can it Reveal the Molecular Sociology of Cells in Atomic Detail? Trends in Cell Biology 26, 825-837.

Bejarano, D.A., Peng, K., Laketa, V., Borner, K., Jost, K.L., Lucic, B., Glass, B., Lusic, M., Mueller, B., and Krausslich, H.G. (2019). HIV-1 nuclear import in macrophages is regulated by CPSF6-capsid interactions at the nuclear pore complex. Elife 8.

Bhattacharya, A., Alam, S.L., Fricke, T., Zadrozny, K., Sedzicki, J., Taylor, A.B., Demeler, B., Pornillos, O., Ganser-Pornillos, B.K., Diaz-Griffero, F., et al. (2014). Structural basis of HIV-1 capsid recognition by PF74 and CPSF6. P Natl Acad Sci USA 111, 18625-18630.

Bichel, K., Price, A.J., Schaller, T., Towers, G.J., Freund, S.M.V., and James, L.C. (2013). HIV-1 capsid undergoes coupled binding and isomerization by the nuclear pore protein NUP358. Retrovirology 10. Briggs, J.A.G., Wilk, T., Welker, R., Krausslich, H.G., and Fuller, S.D. (2003). Structural organization of authentic, mature HIV-1 virions and cores. Embo Journal 22, 1707-1715.

Brohawn, S.G., Partridge, J.R., Whittle, J.R.R., and Schwartz, T.U. (2009). The Nuclear Pore Complex Has Entered the Atomic Age. Structure 17, 1156-1168.

Buffone, C., Martinez-Lopez, A., Fricke, T., Opp, S., Severgnini, M., Cifola, I., Petiti, L., Frabetti, S., Skorupka, K., Zadrozny, K.K., et al. (2018). Nup153 Unlocks the Nuclear Pore Complex for HIV-1 Nuclear Translocation in Nondividing Cells. J Virol 92.

Burdick, R.C., Li, C.L., Munshi, M., Rawson, J.M.O., Nagashima, K., Hu, W.S., and Pathak, V.K. (2020). HIV-1 uncoats in the nucleus near sites of integration. Proceedings of the National Academy of Sciences of the United States of America 117, 5486-5493.

Busnadiego, I., Kane, M., Rihn, S.J., Preugschas, H.F., Hughes, J., Blanco-Melo, D., Strouvelle, V.P., Zang, T.M., Willett, B.J., Boutell, C., et al. (2014). Host and Viral Determinants of Mx2 Antiretroviral Activity. Journal of Virology 88, 7738-7752.

Chen, N.Y., Zhou, L.H., Gane, P.J., Opp, S., Ball, N.J., Nicastro, G., Zufferey, M., Buffone, C., Luban, J., Selwood, D., et al. (2016). HIV-1 capsid is involved in post-nuclear entry steps. Retrovirology 13.

Chin, C.R., Perreira, J.M., Savidis, G., Portmann, J.M., Aker, A.M., Feeley, E.M., Smith, M.C., and Brass, A.L. (2015). Direct Visualization of HIV-1 Replication Intermediates Shows that Capsid and CPSF6 Modulate HIV-1 Intra-nuclear Invasion and Integration. Cell Reports 13, 1717-1731.

Chodera, J.D., and Noé, F. (2014). Markov state models of biomolecular conformational dynamics. Curr Opin Struc Biol 25, 135-144.

Chodera, J.D., and Noe, F. (2014). Markov state models of biomolecular conformational dynamics. Current Opinion in Structural Biology 25, 135-144.

Cook, A., Bono, F., Jinek, M., and Conti, E. (2007). Structural biology of nucleocytoplasmic transport. Annu Rev Biochem 76, 647-671.

Dharan, A., Bachmann, N., Talley, S., Zwikelmaier, V., and Campbell, E.M. (2020). Nuclear pore blockade reveals that HIV-1 completes reverse transcription and uncoating in the nucleus. Nature Microbiology.

Di Nunzio, F., Danckaert, A., Fricke, T., Perez, P., Fernandez, J., Perret, E., Roux, P., Shorte, S., Charneau, P., Diaz-Griffero, F., et al. (2012). Human Nucleoporins Promote HIV-1 Docking at the Nuclear Pore, 
Nuclear Import and Integration. Plos One 7.

Di Nunzio, F., Fricke, T., Miccio, A., Valle-Casuso, J.C., Perez, P., Souque, P., Rizzi, E., Severgnini, M., Mavilio, F., Charneau, P., et al. (2013). Nup153 and Nup98 bind the HIV-1 core and contribute to the early steps of HIV-1 replication. Retrovirology 10, S38-S38.

Dick, R.A., Mallery, D.L., Vogt, V.M., and James, L.C. (2018). IP6 Regulation of HIV Capsid Assembly, Stability, and Uncoating. Viruses-Basel 10.

Dietz, H., Douglas, S.M., and Shih, W.M. (2009). Folding DNA into Twisted and Curved Nanoscale Shapes. Science 325, 725-730.

Duheron, V., Chatel, G., Sauder, U., Oliveri, V., and Fahrenkrog, B. (2014). Structural characterization of altered nucleoporin Nup153 expression in human cells by thin-section electron microscopy. Nucleus 5 , 601-612.

Eswar, N., Webb, B., Marti-Renom, M.A., Madhusudhan, M.S., Eramian, D., Shen, M.Y., Pieper, U., and Sali, A. (2006). Comparative protein structure modeling using Modeller. Curr Protoc Bioinformatics Chapter 5, Unit-5 6.

Fahrenkrog, B., Maco, B., Fager, A.M., Koser, J., Sauder, U., Ullman, K.S., and Aebi, U. (2002). Domainspecific antibodies reveal multiple-site topology of Nup153 within the nuclear pore complex. Journal of Structural Biology 140, 254-267.

Fay, N., and Pante, N. (2015). Nuclear entry of DNA viruses. Frontiers in Microbiology 6.

Feldherr, C.M., Akin, D., and Cohen, R.J. (2001). Regulation of functional nuclear pore size in fibroblasts. Journal of Cell Science 114, 4621-4627.

Fernandez, J., Machado, A.K., Lyonnais, S., Chamontin, C., Gartner, K., Leger, T., Henriquet, C., Garcia, C., Portilho, D.M., Pugniere, M., et al. (2019). Transportin-1 binds to the HIV-1 capsid via a nuclear localization signal and triggers uncoating. Nature Microbiology 4, 1840-+.

Fisher, P.D.E., Shen, Q., Akpinar, B., Davis, L.K., Chun, K.K.H., Baddeley, D., Saric, A., Melia, T.J., Hoogenboom, B.W., Lin, C.X., et al. (2018). A Programmable DNA Origami Platform for Organizing Intrinsically Disordered Nucleoporins within Nanopore Confinement. Acs Nano 12, 1508-1518.

Francis, A.C., Marin, M., Singh, P.K., Achuthan, V., Prellberg, M.J., Palermino-Rowland, K., Lan, S.Y., Tedbury, P.R., Sarafianos, S.G., Engelman, A.N., et al. (2020). HIV-1 replication complexes accumulate in nuclear speckles and integrate into speckle-associated genomic domains. Nature Communications 11.

Gallay, P., Hope, T., Chin, D., and Trono, D. (1997). HIV-1 infection of nondividing cells through the recognition of integrase by the importin/karyopherin pathway. Proceedings of the National Academy of Sciences of the United States of America 94, 9825-9830.

Gallucci, L., and Kann, M. (2017). Nuclear Import of Hepatitis B Virus Capsids and Genome. VirusesBasel 9.

Ganser, B.K., Li, S., Klishko, V.Y., Finch, J.T., and Sundquist, W.I. (1999). Assembly and analysis of conical models for the HIV-1 core. Science 283, 80-83.

Hofer, U. (2020). Entering and breaking for HIV? Nature Reviews Microbiology 18, 264.

Huang, J., Rauscher, S., Nawrocki, G., Ran, T., Feig, M., de Groot, B.L., Grubmuller, H., and MacKerell, A.D., Jr. (2017). CHARMM36m: an improved force field for folded and intrinsically disordered proteins. Nat Methods 14, 71-73.

Hulme, A.E., Kelley, Z., Okocha, E.A., and Hope, T.J. (2015). Identification of Capsid Mutations That Alter the Rate of HIV-1 Uncoating in Infected Cells. Journal of Virology 89, 643-651.

Hulme, A.E., Perez, O., and Hope, T.J. (2011). Complementary assays reveal a relationship between 
HIV-1 uncoating and reverse transcription. P Natl Acad Sci USA 108, 9975-9980.

Husic, B.E., and Pande, V.S. (2018a). Markov State Models: From an Art to a Science. Journal of the American Chemical Society 140, 2386-2396.

Husic, B.E., and Pande, V.S. (2018b). Markov State Models: From an Art to a Science. J Am Chem Soc 140, 2386-2396.

Jang, S., Cook, N.J., Pye, V.E., Bedwell, G.J., Dudek, A.M., Singh, P.K., Cherepanov, P., and Engelman, A.N. (2019). Differential role for phosphorylation in alternative polyadenylation function versus nuclear import of SR-like protein CPSF6. Nucleic Acids Research 47, 4663-4683.

Jorgensen, W.L., and Jenson, C. (1998). Temperature dependence of TIP3P, SPC, and TIP4P water from NPT Monte Carlo simulations: Seeking temperatures of maximum density. Journal of Computational Chemistry 19, 1179-1186.

Kane, M., Rebensburg, S.V., Takata, M.A., Zang, T.M., Yamashita, M., Kvaratskhelia, M., and Bieniasz, P.D. (2018). Nuclear pore heterogeneity influences HIV-1 infection and the antiviral activity of MX2. Elife 7.

Kane, M., Yadav, S.S., Bitzegeio, J., Kutluay, S.B., Zang, T., Wilson, S.J., Schoggins, J.W., Rice, C.M., Yamashita, M., Hatziioannou, T., et al. (2013). MX2 is an interferon-induced inhibitor of HIV-1 infection. Nature 502, 563-+.

Ketterer, P., Ananth, A.N., Trip, D.S.L., Mishra, A., Bertosin, E., Ganji, M., van der Torre, J., Onck, P., Dietz, H., and Dekker, C. (2018). DNA origami scaffold for studying intrinsically disordered proteins of the nuclear pore complex. Nature Communications 9.

Kim, K., Dauphin, A., Komurlu, S., McCauley, S.M., Yurkovetskiy, L., Carbone, C., Diehl, W.E., StrambioDe-Castillia, C., Campbell, E.M., and Luban, J. (2019). Cyclophilin A protects HIV-1 from restriction by human TRIM5 alpha. Nature Microbiology 4, 2044-+.

King, M.C., Raposo, G., and Lemmon, M.A. (2004). Inhibition of nuclear import and cell-cycle progression by mutated forms of the dynamin-like GTPase MxB. Proceedings of the National Academy of Sciences of the United States of America 101, 8957-8962.

Koh, Y., Wu, X.L., Ferris, A.L., Matreyek, K.A., Smith, S.J., Lee, K., KewalRamani, V.N., Hughes, S.H., and Engelman, A. (2013). Differential Effects of Human Immunodeficiency Virus Type 1 Capsid and Cellular Factors Nucleoporin 153 and LEDGF/p75 on the Efficiency and Specificity of Viral DNA Integration. Journal of Virology 87, 648-658.

Li, S., Hill, C.P., Sundquist, W.I., and Finch, J.T. (2000). Image reconstructions of helical assemblies of the HIV-1CA protein. Nature 407, 409-413.

Lin, C.X., Perrault, S.D., Kwak, M., Graf, F., and Shih, W.M. (2013). Purification of DNA-origami nanostructures by rate-zonal centrifugation. Nucleic Acids Research 41.

Lin, D.H., and Hoelz, A. (2019a). The Structure of the Nuclear Pore Complex (An Update). Annu Rev Biochem 88, 725-783.

Lin, D.H., and Hoelz, A. (2019b). The Structure of the Nuclear Pore Complex (An Update). Annual Review of Biochemistry, Vol 88 88, 725-783.

Lippert, R.A., Predescu, C., lerardi, D.J., Mackenzie, K.M., Eastwood, M.P., Dror, R.O., and Shaw, D.E. (2013). Accurate and efficient integration for molecular dynamics simulations at constant temperature and pressure. J Chem Phys 139, 164106.

Liu, J., Sadre-Marandi, F., Tavener, S., and Chen, C. (2015). Curvature Concentrations on the HIV-1

Capsid. Computational and Mathematical Biophysics.

Lopez, C.S., Eccles, J.D., Still, A., Sloan, R.E., Barklis, R.L., Tsagli, S.M., and Barklis, E. (2011). 
Determinants of the HIV-1 core assembly pathway. Virology 417, 137-146.

Lu, R., Limon, A., Devroe, E., Silver, P.A., Cherepanov, P., and Engelman, A. (2004). Class II integrase mutants with changes in putative nuclear localization signals are primarily blocked at a postnuclear entry step of human immunodeficiency virus type 1 replication. Journal of Virology 78, 12735-12746. Lusic, M., and Siliciano, R.F. (2017). Nuclear landscape of HIV-1 infection and integration. Nature Reviews Microbiology 15, 69-82.

MacQueen, J. (1967). Some methods for classification and analysis of multivariate observations. Paper presented at: Proceedings of the fifth Berkeley symposium on mathematical statistics and probability (Oakland, CA, USA).

Maertens, G.N., Cook, N.J., Wang, W.F., Hare, S., Gupta, S.S., Oztop, I., Lee, K.E., Pye, V.E., Cosnefroy, O., Snijders, A.P., et al. (2014). Structural basis for nuclear import of splicing factors by human Transportin 3. Proceedings of the National Academy of Sciences of the United States of America 111, 2728-2733.

Mahamid, J., Pfeffer, S., Schaffer, M., Villa, E., Danev, R., Cuellar, L.K., Forster, F., Hyman, A.A., Plitzko, J.M., and Baumeister, W. (2016). Visualizing the molecular sociology at the HeLa cell nuclear periphery. Science 351, 969-972.

Matreyek, K.A., and Engelman, A. (2011). The Requirement for Nucleoporin NUP153 during Human Immunodeficiency Virus Type 1 Infection Is Determined by the Viral Capsid. Journal of Virology 85, 7818-7827.

Matreyek, K.A., Yucel, S.S., Li, X., and Engelman, A. (2013). Nucleoporin NUP153 Phenylalanine-Glycine Motifs Engage a Common Binding Pocket within the HIV-1 Capsid Protein to Mediate Lentiviral Infectivity. Plos Pathog 9.

Mattei, S., Glass, B., Hagen, W.J.H., Krausslich, H.G., and Briggs, J.A.G. (2016). The structure and flexibility of conical HIV-1 capsids determined within intact virions. Science 354, 1434-1437. Ning, J.Y., Zhong, Z., Fischer, D.K., Harris, G., Watkins, S.C., Ambrose, Z., and Zhang, P.J. (2018). Truncated CPSF6 Forms Higher-Order Complexes That Bind and Disrupt HIV-1 Capsid. Journal of Virology 92.

Noe, F., Schutte, C., Vanden-Eijnden, E., Reich, L., and Weikl, T.R. (2009). Constructing the equilibrium ensemble of folding pathways from short off-equilibrium simulations. Proc Natl Acad Sci U S A 106, 19011-19016.

Ori, A., Banterle, N., Iskar, M., Andres-Pons, A., Escher, C., Bui, H.K., Sparks, L., Solis-Mezarino, V., Rinner, O., Bork, P., et al. (2013). Cell type-specific nuclear pores: a case in point for contextdependent stoichiometry of molecular machines. Molecular Systems Biology 9.

Pérez-Hernández, G., Paul, F., Giorgino, T., Fabritiis, G.D., and Noé, F. (2013). Identification of slow molecular order parameters for Markov model construction. The Journal of Chemical Physics 139, 015102.

Pauwels, R., Declercq, E., Desmyter, J., Balzarini, J., Goubau, P., Herdewijn, P., Vanderhaeghe, H., and Vandeputte, M. (1987). Sensitive and Rapid Assay on Mt-4 Cells for Detection of Antiviral Compounds against the Aids Virus. Journal of Virological Methods 16, 171-185.

Phillips, J.C., Braun, R., Wang, W., Gumbart, J., Tajkhorshid, E., Villa, E., Chipot, C., Skeel, R.D., Kale, L., and Schulten, K. (2005). Scalable molecular dynamics with NAMD. J Comput Chem 26, 1781-1802.

Pornillos, O., Ganser-Pornillos, B.K., Kelly, B.N., Hua, Y.Z., Whitby, F.G., Stout, C.D., Sundquist, W.I., Hill, C.P., and Yeager, M. (2009). X-Ray Structures of the Hexameric Building Block of the HIV Capsid. Cell $137,1282-1292$. 
Pornillos, O., Ganser-Pornillos, B.K., and Yeager, M. (2011). Atomic-level modelling of the HIV capsid. Nature 469, 424-+.

Price, A.J., Fletcher, A.J., Schaller, T., Elliott, T., Lee, K., KewalRamani, V.N., Chin, J.W., Towers, G.J., and James, L.C. (2012). CPSF6 Defines a Conserved Capsid Interface that Modulates HIV-1 Replication. Plos Pathog 8.

Price, A.J., Jacques, D.A., McEwan, W.A., Fletcher, A.J., Essig, S., Chin, J.W., Halambage, U.D., Aiken, C., and James, L.C. (2014). Host Cofactors and Pharmacologic Ligands Share an Essential Interface in HIV-1 Capsid That Is Lost upon Disassembly. Plos Pathogens 10.

Prinz, J.-H., Wu, H., Sarich, M., Keller, B., Senne, M., Held, M., Chodera, J.D., Schütte, C., and Noé, F. (2011). Markov models of molecular kinetics: Generation and validation. The Journal of Chemical Physics 134, 174105.

Röblitz, S., and Weber, M. (2013). Fuzzy spectral clustering by PCCA+: application to Markov state models and data classification. Advances in Data Analysis and Classification 7, 147-179.

Rasaiyaah, J., Tan, C.P., Fletcher, A.J., Price, A.J., Blondeau, C., Hilditch, L., Jacques, D.A., Selwood, D.L., James, L.C., Noursadeghi, M., et al. (2013). HIV-1 evades innate immune recognition through specific cofactor recruitment. Nature 503, 402-+.

Rothemund, P.W.K. (2006). Folding DNA to create nanoscale shapes and patterns. Nature 440, $297-$

302.

Schaller, T., Ocwieja, K.E., Rasaiyaah, J., Price, A.J., Brady, T.L., Roth, S.L., Hue, S., Fletcher, A.J., Lee, K., KewalRamani, V.N., et al. (2011). HIV-1 Capsid-Cyclophilin Interactions Determine Nuclear Import Pathway, Integration Targeting and Replication Efficiency. Plos Pathogens 7.

Scherer, M.K., Trendelkamp-Schroer, B., Paul, F., Perez-Hernandez, G., Hoffmann, M., Plattner, N., Wehmeyer, C., Prinz, J.H., and Noe, F. (2015). PyEMMA 2: A Software Package for Estimation, Validation, and Analysis of Markov Models. J Chem Theory Comput 11, 5525-5542.

Schmitz, A., Schwarz, A., Foss, M., Zhou, L.X., Rabe, B., Hoellenriegel, J., Stoeber, M., Pante, N., and Kann, M. (2010). Nucleoporin 153 Arrests the Nuclear Import of Hepatitis B Virus Capsids in the Nuclear Basket. Plos Pathogens 6.

Seeman, N.C., and Sleiman, H.F. (2018). DNA nanotechnology. Nature Reviews Materials 3.

Serrao, E., Cherepanov, P., and Engelman, A.N. (2016). Amplification, Next-generation Sequencing, and Genomic DNA Mapping of Retroviral Integration Sites. Jove-Journal of Visualized Experiments.

Shah, V.B., and Aiken, C. (2011). In vitro Uncoating of HIV-1 Cores. Jove-Journal of Visualized Experiments.

Shan, Y., Klepeis, J.L., Eastwood, M.P., Dror, R.O., and Shaw, D.E. (2005). Gaussian split Ewald: A fast Ewald mesh method for molecular simulation. J Chem Phys 122, 54101.

Shaw, D.E., Grossman, J.P., Bank, J.A., Batson, B., Butts, J.A., Chao, J.C., Deneroff, M.M., Dror, R.O., Even, A., Fenton, C.H., et al. (2014). Anton 2: raising the bar for performance and programmability in a special-purpose molecular dynamics supercomputer. In Proceedings of the International Conference for High Performance Computing, Networking, Storage and Analysis (New Orleans, Louisana: IEEE Press), pp. 41-53.

Shen, M.-y., and Sali, A. (2006). Statistical potential for assessment and prediction of protein structures. Protein Science 15, 2507-2524.

Shun, M.C., Raghavendra, N.K., Vandegraaff, N., Daigle, J.E., Hughes, S., Kellam, P., Cherepanov, P., and Engelman, A. (2007). LEDGF/p75 functions downstream from preintegration complex formation to effect gene-specific HIV-1 integration. Genes \& Development 21, 1767-1778. 
Smaga, S.S., Xu, C.Y., Summers, B.J., Digianantonio, K.M., Perilla, J.R., and Xiong, Y. (2019). MxB Restricts HIV-1 by Targeting the Tri-hexamer Interface of the Viral Capsid. Structure 27, 1234-+. Sowd, G.A., Serrao, E., Wang, H., Wang, W.F., Fadel, H.J., Poeschla, E.M., and Engelman, A.N. (2016). A critical role for alternative polyadenylation factor CPSF6 in targeting HIV-1 integration to transcriptionally active chromatin. Proceedings of the National Academy of Sciences of the United States of America 113, E1054-E1063.

Strambio-De-Castillia, C., Niepel, M., and Rout, M.P. (2010). The nuclear pore complex: bridging nuclear transport and gene regulation. Nature Reviews Molecular Cell Biology 11, 490-501. Summers, B.J., Digianantonio, K.M., Smaga, S.S., Huang, P.T., Zhou, K.F., Gerber, E.E., Wang, W., and Xiong, Y. (2019). Modular HIV-1 Capsid Assemblies Reveal Diverse Host-Capsid Recognition Mechanisms. Cell Host \& Microbe 26, 203-+.

Valle-Casuso, J.C., Di Nunzio, F., Yang, Y., Reszka, N., Lienlaf, M., Arhel, N., Perez, P., Brass, A.L., and Diaz-Griffero, F. (2012). TNPO3 Is Required for HIV-1 Replication after Nuclear Import but prior to Integration and Binds the HIV-1 Core. Journal of Virology 86, 5931-5936.

von Appen, A., Kosinski, J., Sparks, L., Ori, A., DiGuilio, A.L., Vollmer, B., Mackmull, M.T., Banterle, N., Parca, L., Kastritis, P., et al. (2015). In situ structural analysis of the human nuclear pore complex. Nature 526, 140-+.

Walther, T.C., Fornerod, M., Pickersgill, H., Goldberg, M., Allen, T., and Mattaj, I.W. (2001). The nucleoporin Nup153 is required for nuclear pore basket formation, Nuclear Pore Complex anchoring and import of a subset of nuclear proteins. Molecular Biology of the Cell 12, 232a-232a.

Wang, W., Cao, S., Zhu, L., and Huang, X. (2018a). Constructing Markov State Models to elucidate the functional conformational changes of complex biomolecules. WIREs Computational Molecular Science 8, e1343.

Wang, W., Cao, S.Q., Zhu, L.Z., and Huang, X.H. (2018b). Constructing Markov State Models to elucidate the functional conformational changes of complex biomolecules. Wiley Interdisciplinary Reviews-Computational Molecular Science 8.

Wehrly, K., and Chesebro, B. (1997). p24 antigen capture assay for quantification of human immunodeficiency virus using readily available inexpensive reagents. Methods-a Companion to Methods in Enzymology 12, 288-293.

Yamashita, M., and Engelman, A.N. (2017). Capsid-Dependent Host Factors in HIV-1 Infection. Trends in Microbiology 25, 741-755.

Zhang, Y.Q., Li, S., Zeng, C., Huang, G.X., Zhu, X.C., Wang, Q.F., Wang, K.P., Zhou, Q., Yan, C.Y., Zhang, W.S., et al. (2020). Molecular architecture of the luminal ring of the Xenopus laevis nuclear pore complex. Cell Research 30, 532-540.

Zhang, Z.Q., He, M.Z., Bai, S.M., Zhang, F., Jiang, J., Zheng, Q.B., Gao, S.Q., Yan, X.D., Li, S.W., Gu, Y., et al. (2018). T=4 Icosahedral HIV-1 Capsid As an Immunogenic Vector for HIV-1 V3 Loop Epitope Display. Viruses-Basel 10.

Zhao, G.P., Ke, D.X., Vu, T., Ahn, J., Shah, V.B., Yang, R.F., Aiken, C., Charlton, L.M., Gronenborn, A.M., and Zhang, P.J. (2011). Rhesus TRIM5 alpha Disrupts the HIV-1 Capsid at the Inter-Hexamer Interfaces. Plos Pathogens 7.

Zimmerli, C.E., Allegretti, M., Rantos, V., Goetz, S.K., Obarska-Kosinska, A., Zagoriy, I., Halavatyi, A., Mahamid, J., Kosinski, J., and Beck, M. (2020). Nuclear pores constrict upon energy depletion. bioRxiv, 2020.2007.2030.228585.

Zuliani-Alvarez, L., and Towers, G.J. (2019). Immunology: Identifying a nuclear passport for HIV. Elife 8. 